\title{
STAG proteins promote cohesin ring loading at R-loops.
}

Hayley Porter ${ }^{1+}$, Yang $\mathrm{Li}^{1+}$, Wazeer Varsally ${ }^{1}$, Maria

Victoria Neguembor ${ }^{2}$, Manuel Beltran ${ }^{3}$, Dubravka Pezic ${ }^{1}$, Laura Martin ${ }^{2}$, Manuel Tavares Cornejo ${ }^{3}$, Amandeep Bhamra ${ }^{4}$, Silvia Surinova ${ }^{4}$, Richard G. Jenner ${ }^{3}$, Maria Pia Cosma ${ }^{2,5,6}$, Suzana Hadjur ${ }^{1 *}$

1 Research Department of Cancer Biology, Cancer Institute, University College London, 72 Huntley Street, London, United Kingdom

2 Centre for Genomic Regulation (CRG), The Barcelona Institute of Science and Technology, 08003 Barcelona, Spain.

3 Regulatory Genomics Group, Cancer Institute, University College London, London WC1E 6BT, United Kingdom

4 Proteomics Research Translational Technology Platform, Cancer Institute, University College London, 72 Huntley Street, London, United Kingdom

5 Universitat Pompeu Fabra (UPF), Dr Aiguader 88, 08003 Barcelona, Spain

6 Institució Catalana de Recerca i Estudis Avançats (ICREA), Pg. Lluís Companys 23, 08010 Barcelona, Spain.

+ These authors contributed equally

*Correspondence: s.hadjur@ucl.ac.uk 


\section{$1 \quad$ ABSTRACT}

3 Most studies of cohesin function consider the Stromalin Antigen (STAG/SA) proteins 4 as core complex members given their ubiquitous interaction with the cohesin ring. 5 Here, we provide functional data to support the notion that the SA subunit is not a 6 mere passenger in this structure, but instead plays a key role in cohesins localization 7 to diverse biological processes and promotes loading of the complex at these sites. 8 We show that in cells acutely depleted for RAD21, SA proteins remain bound to 9 chromatin and interact with CTCF, as well as a wide range of RNA binding proteins 10 involved in multiple RNA processing mechanisms. Accordingly, SA proteins interact 11 with RNA and are localised to endogenous R-loops where they act to suppress R-loop 12 formation. Our results place SA proteins on chromatin upstream of the cohesin 13 complex and reveal a role for SA in cohesin loading at R-loops which is independent 14 of NIPBL, the canonical cohesin loader. We propose that SA takes advantage of this 15 structural R-loop platform to link cohesin loading and chromatin structure with diverse 16 genome functions. Since SA proteins are pan-cancer targets, and R-loops play an 17 increasingly prevalent role in cancer biology, our results have important implications 18 for the mechanistic understanding of SA proteins in cancer and disease.

\section{KEY WORDS}

24 Cohesin, STAG proteins, R-loops, genome. 


\section{INTRODUCTION}

26 Cohesin complexes are master regulators of chromosome structure in interphase and

27 mitosis. Accordingly, mutations of cohesin subunits leads to changes in cellular 28 identity, both during development and aberrantly in cancer ${ }^{1-3}$. A prevailing model is that cohesin contributes to cell identity changes in large part by dynamically regulating genome organization and mediating communication between distal regulatory elements ${ }^{4-10}$. Our understanding of how cohesin's component parts contribute to its functions and where cohesin becomes associated to chromatin in order to perform its critical roles in spatial genome organization is incomplete.

Most studies of cohesin function consider the Stromalin Antigen (STAG/SA) proteins as core complex members given their ubiquitous interaction with the tripartite cohesin ring (composed of SMC1, SMC3 and SCC1/RAD21). Rarely is the SA subunit considered for its roles outside of cohesin, even though it is the subunit most commonly mutated across a wide spectrum of cancers ${ }^{1,11,12}$. SA proteins play a role in cohesin's association with DNA ${ }^{13,14}$. The yeast SA orthologue is critical for efficient association of cohesin with DNA and its ATPase activation ${ }^{13,14}$. Recent crystallization studies of cohesin in complex with its canonical loader NIPBL ${ }^{15}$, suggest that NIPBL and SA together wrap around both the cohesin ring and DNA to position and entrap DNA ${ }^{16-18}$, implying a role for SA in the initial recruitment of cohesin to DNA alongside NIPBL. Further, SA proteins bridge the interaction between cohesin and CTCF ${ }^{16,19,20}$, and also bridge interactions with specific nucleic acid structures in vitro. SA1 binds to AT-rich telomeric sequences ${ }^{21,22}$ and SA2 displays sequence-independent affinity for particular DNA structures commonly found at sites of repair, recombination, and replication ${ }^{23}$. Consistent with this, results in yeast implicate non-canonical DNA structures in cohesin loading in S-phase. In vitro experiments show that cohesin captures the second strand of DNA via a single-strand intermediate ${ }^{24}$, and chromatid cohesion is impaired by de-stabilisation of single-strand DNA intermediates during replication ${ }^{25}$. Together, this implicates SA proteins in playing a regulatory role in guiding or stabilising cohesin localisations.

During transcription, the elongating nascent RNA can hybridise to the template strand of the upstream DNA and form an R-loop, which is an intermediate RNA:DNA hybrid conformation with a displaced single strand of DNA (Richardson, 1975; Roy 
and Lieber, 2009; El Hage et al., 2010; Roy et al., 2010). A multitude of processes have been linked to R-loop stability and metabolism. For example, proper cotranscriptional RNA processing, splicing, and messenger ribonucleoprotein (mRNP) assembly counteract R-loop formation (Li and Manley, 2005; Teloni et al., 2019). Rloop structures have also been shown to regulate transcription of both mRNA and rRNAs by recruitment of transcription factors, displacement of nucleosomes, and preservation of open chromatin (Dunn and Griffith, 1980; Powell et al., 2013; BoqueSastre et al., 2015). Hence, like at the replication fork, sites of active transcription accumulate non-canonical nucleic acid structures.

We set out to investigate the nature of the association between SA proteins and CTCF. We discovered that far from being 'passengers' in the cohesin complex, SA proteins perform critical roles in their own right, directing cohesin's localization and loading to chromatin. In cells acutely depleted of RAD21, SA proteins remain associated with chromatin and CTCF where they are enriched at 3D clustered sites of active chromatin. Moreover, we identify cohesin-independent binding of SA1 to numerous proteins involved in RNA processing, ribosome biogenesis, and translation. Consistent with this, SA1 and SA2 interact with RNA and non-canonical nucleic acid structures in the form of R-loops where SA1 acts to suppress R-loop formation. Importantly, SA proteins are required for loading of cohesin to chromatin in cells deficient for NIPBL, and loading is enhanced by modulating the levels of R-loop structures. Our results highlight a central role for SA proteins in cohesin biology. Through their diverse interactions with proteins, RNA and DNA, SA proteins act as the 'seed' for cohesin loading to chromatin. Finally, the interaction of cohesin-independent SA proteins with nucleolar and RNA processing factors, opens up a new understanding of how cohesin mis-regulation can impact disease development that moves us beyond its control of gene expression regulation. 


\section{SA interacts with CTCF on chromatin in the absence of the cohesin trimer.}

To determine how CTCF and cohesin assemble on chromatin, we used human HCT116 cells engineered to carry a miniAID tag (mAID) fused to monomeric Clover (mClover) at the endogenous Rad21 locus and OsTIR1 under the control of CMV (herein RAD2 $\left.1^{\mathrm{mAC}}\right)^{26}$. RAD2 $1^{\mathrm{mAC}}$ cells were cultured in control conditions (ethanol) or in the presence of auxin (IAA) to induce rapid RAD21 degradation. We used immunofluorescence (IF) to monitor the levels of mClover, SA1, SA2 and CTCF (Fig.1a, b, S1a). While acute IAA treatment robustly reduced mClover levels by over $83 \%$ compared to control cells, SA2 levels were reduced by $63 \%$ ( $p=4.7 \mathrm{E}-76)$, and SA1 was only reduced by $29 \%(p=7.9 E-12)$ (Fig.1b). We also observed a variable effect on CTCF in the absence of RAD21 (reduced mean value between 7-24\%). The retention of SA proteins on chromatin despite the degradation of RAD21 was surprising given the fact that they are considered to be part of a stable biochemical complex.

We sought to validate these observations using an orthogonal technique and to establish whether the residual SA proteins retained the capacity to directly interact with CTCF. We prepared chromatin extracts from RAD2 $1^{\mathrm{mAC}}$ cells treated with ethanol or IAA and established a chromatin co-immunoprecipitation (coIP) protocol to probe the interactions between SA proteins, cohesin core subunits and CTCF. Both SA1 and SA2 interacted with RAD21 and CTCF in control cells as expected ${ }^{27,28}$, with notable differences in their preferred interactions (Fig.1c). SA2 more strongly enriched RAD21 while the SA1-CTCF interaction was significantly stronger than SA2-CTCF (Fig.1c). Upon RAD21 degradation, we again observed a stronger effect on chromatinbound SA2 levels compared to SA1, implying stable binding of SA2 to chromatin is more sensitive to cohesin loss than SA1. Not only did the residual SA proteins retain

110 their ability to interact with CTCF in the absence of Rad21, but the interactions

111 between SA1 and CTCF were further enhanced (Fig.1c). Reciprocal colPs with CTCF

112 confirmed the CTCF-SA interactions in RAD21-depleted cells (Fig.1d). These results

113 were validated in a second cell line and upon siRNA-mediated knockdown of SMC3, 114 confirming the results (Fig S1b). 
We performed two-color Stochastic Optical Reconstruction Microscopy

116 (STORM) to further assess the nuclear distribution and colocalization of SA1, SA2 and

117 CTCF with nanometric resolution in RAD21-degraded cells. Upon IAA treatment, we 118 observed a decreased density of detected SA1, SA2 and CTCF in two analyzed clones

119 (Fig.1e, f, S1c, d), suggesting that RAD21 degradation affects the stability of CTCF in 120 addition to SA proteins. As we observed by conventional confocal microscopy, SA2 121 localizations were more affected than SA1 (mean density reduction in SA1, 32\% vs 122 SA2, 42\%). Accordingly, SA1, SA2 and CTCF clusters were more sparsely distributed 123 across the nucleus upon RAD21 degradation as quantified by nearest neighbor 124 distance (NND) analysis of protein clusters (Fig.1g). This analysis also revealed a 125 higher density of SA1 and CTCF clusters compared to SA2, with shorter distances 126 between clusters, even in ethanol conditions (Fig.1g). To further confirm that SA and 127 CTCF were still co-localised in IAA conditions, we analyzed the relative distribution of 128 SA clusters to CTCF clusters by analyzing the NND distribution between SA1 and 129 CTCF, and SA2 and CTCF. NND showed that the association between SA1 and SA2 130 with CTCF is maintained upon RAD21 degradation as compared to both the control 131 cells and to a simulation of randomly-distributed protein clusters at the same density 132 (Fig. 1h). Interestingly, while the probability of SA1 at CTCF is only modestly affected 133 in IAA conditions, supporting their continued co-localization, SA2 at CTCF is more 134 affected in IAA treated cells, in line with results indicating that SA2 levels are more 135 affected than SA1 when cohesin is depleted. Together, our results confirm the 136 maintained interaction and localization patterns of SA proteins with CTCF and reveal 137 a difference in SA paralogue stability in the absence of the core cohesin trimer.

139 Cohesin-independent SA proteins are localised at clustered regions in 3D.

140 Previous analyses of the contribution of SA proteins to genome organization ${ }^{7,9}$ were 141 performed in cells containing cohesin rings, possibly obscuring a functional role for SA 142 proteins themselves in genome organization. To determine if cohesin-independent SA 143 proteins may function at unique locations in the genome, we investigated whether the 144 residual SA-CTCF complexes (herein, SA-CTCF ${ }^{\triangle \mathrm{Coh}}$ ) in IAA-treated RAD2 $1^{\mathrm{mAC}}$ cells

145 (Fig.1c, d) occupied the same chromatin locations as in control cells. Using chromatin 146 immunopreciptation followed by sequencing (ChIP-seq), we determined the binding 
147 profiles of CTCF, SA1, SA2, RAD21 and SMC3 in RAD21 ${ }^{\mathrm{mAC}}$ cells treated with ethanol 148 or IAA. Pairwise comparisons of CTCF ChIP-seq with RAD21 or SA in control

149 RAD21 ${ }^{\mathrm{mAC}}$ cells revealed the expected overlap in binding sites (Fig.1i, S1e). In 150 contrast, both global and CTCF-overlapping RAD21 and SMC3 ChIP-seq signals were 151 dramatically lost in IAA-treated cells (Fig.1i, S1e). In agreement with our microscopy 152 and biochemistry results, we detected residual SA1 and SA2 binding sites in IAA153 treated cells which retained a substantial overlap with CTCF (Fig.1i, S1e). We confirmed that the sites co-occupied by CTCF and SA proteins in RAD21-depleted cells were previously bound in control conditions, indicating that CTCF and SA maintain occupancy at their canonical binding sites in the absence of RAD21. This suggests that SA interaction with CTCF in the absence of the cohesin ring is a step in normal cohesin activity.

While depletion of cohesin results in a dramatic loss of Topologically Associated Domain (TAD) structure ${ }^{8}$, the frequency of long-range inter-TAD, intracompartment contacts (LRC) is increased ${ }^{5,8}$, and enriched for CTCF ${ }^{5}$ or active enhancers $^{8}$. To determine whether residual, chromatin-bound SA could be associated with LRCs in the absence of RAD21, we re-analysed Hi-C data from control and IAAtreated RAD2 $1^{\mathrm{mAC}}$ cells ${ }^{8}$. We quantified all contacts within two different scales of genome organization; local TAD topology (100k-1Mb) and clustered LRCs (1-5Mb) (Fig.1j). As previously shown, local TAD contacts are lost and clustered LRCs are enriched in IAA conditions (Fig.1j). When we probed the Hi-C datasets for contacts containing the residual SA-CTCF ${ }^{\Delta C o h}$ binding sites, we observed a further enrichment in IAA conditions (Fig.1j, bottom row), indicating that SA-CTCF ${ }^{\Delta C o h}$ are enriched at the clustered LRCs formed when cells are depleted of cohesin and thus implicating them in 3D structural configurations. Finally, using ChromHMM, we discovered that SA$\mathrm{CTCF}^{\Delta \mathrm{Coh}}$ sites are characterised by active chromatin and enhancers (Fig.S1f). Our results suggest that cohesin-independent SA, either with CTCF or alone, may itself contribute to large-scale arrangement of active chromatin and regulatory features in 3D space. 
179 SA interacts with diverse 'CES-binding proteins' in RAD21-depleted cells.

180 SA proteins contain a highly conserved domain termed the 'stromalin conservative 181 domain' ${ }^{14,29}$, or the 'conserved essential surface' (CES). Structural analysis of CTCF182 SA2-SCC1(RAD21) has recently shown that FGF (F/YxF) motifs in the N-terminus of 183 CTCF bind to the CES on the SA2-SCC1 sub-complex, forming a tripartite interaction 184 patch ${ }^{16}$. Furthermore, the authors identified an FGF-like motif in additional cohesin 185 regulators and showed that a consensus motif could be used to predict interaction with 186 additional chromatin proteins. Thus, we investigated whether SA could associate with 187 other FGF-motif containing proteins in native and IAA conditions in cells. We 188 performed chromatin IP with SA1 and SA2 in ethanol and IAA and probed for 189 interaction with CTCF, and three additional FGF-motif containing proteins, CHD6, 190 MCM3 and HNRNPUL2 (Fig.2a, S2a). As with CTCF, all of the proteins directly 191 interacted with SA1 in RAD21-control cells and furthermore their interaction with SA1 192 was enriched upon RAD21-degradation. Interestingly, despite SA2 also containing the conserved 'CES' domain, the FGF-motif proteins did not interact with SA2 as strongly

194 (Fig.2a), pointing to an additional element which functions to stabilise SA with FGF195 containing proteins in vivo. These results revealed that SA can interact with proteins 196 beyond just CTCF in the absence of cohesin, indicating a need to re-evaluate the role 197 of SA in cohesin activity and consider possible novel functions for SA proteins.

199 SA1 interacts with a diverse group of proteins in the absence of cohesin.

200 To delineate novel protein binding partners and putative biological functions of SA1, 201 we optimised our chromatin-bound, endogenous SA1 co-IP protocol to be compatible 202 with mass-spectrometry (IP-MS) and used this to comprehensively characterize the 203 SA1 protein-protein interaction (PPI) network in control or RAD21-degraded 204 RAD2 $1^{\mathrm{mAC}}$ cells. Three biological replicate sets were prepared from RAD21 ${ }^{\mathrm{mAC}}$ cells 205 that were either untreated (UT) or treated with IAA (IAA) and processed for IP with 206 both SA1 and IgG antibodies. In parallel, RAD $21^{\mathrm{mAC}}$ cells were also treated with 207 scrambled siRNAs or with siRNA to SA1 to confirm the specificity of putative 208 interactors (Fig. S2b). Immunoprecipitated proteins were in-gel Trypsin digested, gel 209 extracted, and identified by liquid chromatography tandem mass spectroscopy (LC- 
MS/MS). SA1 peptides were robustly detected in all UT and siCON samples and never detected in IgG controls, validating the specificity of the antibody.

We identified 1282 unique proteins that co-purified with SA1 with a False Discovery Rate $<1 \%$. After filtering steps (methods), we used a pairwise analysis of

214 IAA vs UT samples to generate a fold-change value for each putative interactor. These

215 candidates were found in at least 2 of the 3 SA1 IP replicates, were changed by at

216 least 1.5-fold compared to UT controls, and sensitive to siSA1, yielding 134 high217 confidence cohesin-independent SA1 (SA1 ${ }^{\Delta \mathrm{Coh}}$ ) interactors (Fig. 2b, Table 1). As 218 expected, core cohesin subunits SMC1A and SMC3 were strongly depleted while no 219 peptides were detected for RAD21 (Fig. 2b). SA1 itself was significantly depleted compared to control cells, as were other cohesin regulators, known to directly interact with SA1, such as PDS5B ${ }^{30}$. In line with the enrichment we observed for the CESbinding proteins in IAA-conditions (Fig. 1C, 2a), the vast majority of the $S A 1^{\triangle \mathrm{C} o h}$ interactors were enriched for binding with SA1 in IAA conditions (117 of 134) (Fig. 2b). interactome and to identify enriched biological processes and molecular functions. This revealed that the $S A 1^{\Delta \mathrm{Coh}} \mathrm{PPI}$ network included gene expression, chromatin, cytoplasmic and RNA binding proteins representing a variety of functionally diverse cellular processes. Among these are processes previously associated with cohesin biology and identified in published cohesin mass-spec experiments ${ }^{31}$, thus validating our approach, such as MCM3 and SWI/SNF components INO80 and SMARCAL1 which are involved in DNA replication and chromatin remodelling, respectively.

232 Similarly, several transcriptional and epigenetic regulators were identified, such as PRC2 component JARID2 and TAF15 and SPTY2D1.

In addition, we identified proteins associated with SA1 in IAA conditions that were involved in functions that have not been previously associated with SA biology (Fig. 2c, d). The most enriched category was RNA processing $\left(p=3.62^{-39}\right)$, and

237 included proteins involved in RNA modification (YTHDC1, ADAR1, FTSJ3); mRNA 238 stabilization and export (SYNCRIP, FMR1); and several RNA splicing regulators

239 (SRSF1, SON). Accordingly, we found a significant enrichment for DNA and RNA 240 helicases $\left(p=3.54^{-08}\right)\left(\mathrm{MCM} 3, \mathrm{DHX}\right.$, more) and RNA binding proteins $\left(p=9.11^{-11}\right)$ 
within which were serveal hnRNP family members (hnRNPU or SAF-A). We also found

242 a highly significant enrichment of proteins associated with Ribosome biogenesis

$243\left(\mathrm{p}=2.20^{-30}\right)$ including both large and small subunit components (RPL5, 17, 29, RPS9);

244 rRNA processing factors (BOP1, NOP56); and components of the snoRNA pathway.

245 Translation was significantly enriched as a biological process $\left(p=1.64^{-06}\right)$, with several

246 cytoplasmic translation regulators also identified as $S A 1^{\Delta \mathrm{Coh}}$ interactors (Fig. 2c, d).

247 Among these are ESYT2 and EIF3B which we identify as FGF-containing proteins that

248 are primarily found in the cytoplasm (Fig. 2d). We validated 8 of the highest-ranking

249 proteins within the enriched functional categories described above by immunoblotting

250 in ethanol and IAA-treated RAD2 $1^{\mathrm{mAC}}$ cells (Fig. 2d). Overall, our results show that

$251 \mathrm{SA}^{\Delta \mathrm{Coh}} \mathrm{PPIs}$ contain not only for transcriptional and epigenetic regulators, but also

252 are predominantly enriched for proteins with roles in nuclear RNA processing and

253 modification, ribogenesis and translation pathways. Accordingly, this suggests that SA

254 may facilitate an aspect of cohesin regulation at a variety of functionally distinct cellular locations through its association with these diverse proteins.

\section{SA proteins directly bind RNA.}

258 Since RNA binding and RNA processing were two of the most enriched categories in

259 the SA1 ${ }^{\Delta \text { Coh }}$ PPI network, we asked whether SA proteins could also bind RNA. We

260 performed CLIP (crosslinking and immunoprecipitation) to determine whether SA

261 proteins directly bind RNA in untreated RAD2 $1^{\mathrm{mAC}}$ cells. We found that both SA1 and

262 SA2 directly bound RNA (Fig.3a). This was evidenced by detection of RNPs of the

263 expected molecular weights, with a smear of trimmed RNA, which was stronger in the

$264+$ UV and +PNK conditions, which increased as the RNasel concentration was reduced

265 and which was lost after siRNA-mediated KD (Fig.3a, S3a). We repeated the

266 experiment in IAA-treated RAD2 $1^{\mathrm{mAC}}$ cells to determine if the SA subunits can directly

267 bind RNA in the absence of cohesin. Although RAD21 depletion reduced SA1 and

268 SA2 stability, the amount of RNA crosslinked to the proteins remained proportional to

269 the amount of SA1 and SA2 protein, demonstrating that cohesin is not required for the

270 interaction of these proteins with RNA in cells (Fig.3b, S3b. Thus, cohesin-

271 independent SA proteins interact with a wide array of RNA binding proteins (RBP) as

272 well as with RNA itself. 


\section{A variable exon in the C-terminus of SA tunes association w RNA binding} proteins.

275 SA1 and SA2 express transcript variants in RAD21 ${ }^{\mathrm{mAC}}$ cells. One such prominent variant arises from the alternative splicing of a single C-terminal exon, exon 31 in SA1 $\left(\mathrm{SA} 1^{\mathrm{e} 1 \Delta}\right.$ ) and exon 32 in SA2 (SA2 $\left.{ }^{\mathrm{e} 32 \Delta}\right)$ (Fig.3c). This has been observed in many cell types, however the significance of this variant is unknown. We re-analysed publicly available RNA-seq datasets for gene expression and alternative splicing. Interestingly, quantification of the splicing profiles using VAST-tools analysis ${ }^{32}$ revealed that the frequency of the e31 or e32 splicing events were dramatically different (Fig.3d). The majority of SA1 mRNAs include e31 (average PSI 97.7\%), while the majority of SA2 mRNAs exclude e32 (average PSI 20.4\%). We confirmed this at the protein level by designing custom esiRNAs to specifically target SA1 e31 or SA2 e32 (Methods). Smartpool (SP) KD reduced the levels of SA1 and SA2 to similar extents compared to scrambled controls ( $87 \%$ and $94 \%$ respectively compared to siRNA control) (Fig. 3e, f). Specific targeting of SA1 e31 led to a reduction of $85 \%$ of SA1 compared to esiRNA control (Fig.3e), while SA2 e32 targeting had a minimal effect on SA2 protein levels compared to its esiRNA control (reduction of $2 \%$ ) (Fig.3f), in line with the PSI data.

These results imply that cells 'tune' the availability of e31/32 domains in SA proteins, prompting us to investigate the nature of these exons to shed light on their potential function. Inspection of the amino acid (aa) sequence of the spliced exons revealed that they encode a highly basic domain within an otherwise acidic $\mathrm{C}$-terminus (Fig.3c). Overall, the SA paralogs are highly homologous, however the $\mathrm{N}$ and $\mathrm{C}$ termini diverge in their aa sequence. Despite this divergence, e31 and e32 have retained their basic properties with a pl similar to histones $(\mathrm{pl}=10.4,9.9$ for e31 and e32, respectively) (Fig.3c). Basic domains act as important regulatory cassettes and can bind nucleic acids. Thus, we investigated whether the alternatively spliced basic exon of SA proteins contributes to the differential association of SA with RNA (Fig.3a,b). We cloned cDNAs from HCT116 cells representing full-length SA2 (SA2 ${ }^{\mathrm{FL}}$ ) and the variant lacking e32 (SA2 $\left.{ }^{\mathrm{e} 32 \Delta}\right)$, tagged them with YFP and expressed them in HCT cells (Fig.S3c). We used the GFP-TRAP system to specifically purify the YFP-tagged isoforms from cells and compared their ability to interact with RNA. While CLIP experiments revealed that the presence of the alternative e32 does not affect the ability of SA2 to interact with RNA (Fig.3g, S3d), however it did reveal bands which 
306 were enriched in the YFP-SA2 ${ }^{\mathrm{FL}}$ CLIP and not observed in the YFP-SA2 ${ }^{\mathrm{e} 2 \Delta}$ samples,

307 revealing a role for the alternative exon in enhanced association of SA2 with RNA

308 binding proteins.

\section{SA proteins bind to endogenous R-loops.}

311 Regulators of RNA processing, such as splicing, modification and export factors, act

312 as regulators of $\mathrm{R}$-loops ${ }^{33}$. In addition, $\mathrm{R}$-loops are found at sites of multiple biological

313 processes including transcription (of both mRNAs and rRNA), DNA replication and

314 DNA repair ${ }^{33}$. Given the fact that many of these processes were enriched in the SA1

315 interactome and our observations that SA proteins can interact with RNA, we reasoned

316 that the diversity of biological processes represented in the SA ${ }^{\triangle \mathrm{C} \text { coh }} \mathrm{PPI}$ network may

317 be reflective of a role for SA proteins in R-loop biology.

318 To address this, we returned to our IP-MS experiment to analyse enrichment of

319 R-loop-associated proteins in our SA1 ${ }^{\Delta \mathrm{Coh}}$ interactome. We overlapped the proteins

320 identified in two independent IP-MS experiments using the R-loop specific antibody,

$321 S 9.6^{34,35}$ to create a custom high-confidence 'R-loop interactome' and then used a

322 hygrometric distribution to determine the significance of this category in the $S A 1^{\Delta C o h}$

323 interactome (methods). Both the custom R-loop interactome as well as the S9.6

324 interactomes from Cristini et al., and Wang et al., were highly over-enriched in the

$325 \mathrm{SA} 1^{\Delta \mathrm{Coh}}$ interactome $\left(\mathrm{FDR}=1.10 \times 10^{-15}, 1.38 \times 10^{-47}\right.$, respectively) (Fig.4a). As an

326 independent validation of these observations, we optimised an S9.6 colP method in

327 RAD21 ${ }^{\mathrm{mAC}}$ cells (Fig.4b, methods). In agreement with published results, we found that

328 S9.6 precipitated with the known R-loop helicases AQR, DHX9, RNase H2 ${ }^{34,36}$ as well

329 as MCM3 and RNA Pol II ${ }^{37}$. Both SA1 and SA2 precipitated with S9.6 (Fig.4b, S3x),

330 indicating a function at R-loops and supporting the observed enrichment of R-loop

331 proteins in the SA1 interactome.

332 To understand the causal relationship between R-loops and SA proteins, and

333 to determine the specificity of S9.6-SA interactions, we used RNase $\mathrm{H} 1$ to selectively

334 degrade the RNA component of RNA:DNA hybrids ${ }^{38}$. We were able to achieve a $335 \sim 30 \%$ reduction in R-loops upon treatment of chromatin lysates with RNase H (Fig.4b,

336 S4a). This reduction was proportional to the observed reduction in colP of SA1 by

337 S9.6 (Fig.4b, S4b). In parallel, we assessed the effect of R-loop degradation on 
338 chromatin-bound SA levels in single cells using confocal microscopy. Treatment of

$339 \mathrm{RAD} 21^{\mathrm{mAC}}$ cells with $\mathrm{RNase} \mathrm{H} 1$ reduces $\mathrm{S} 9.6$ staining by $>50 \%$ of untreated controls

340 (Fig.4c, d). In agreement with the S9.6 colP results, mean levels of SA1 and SA2 were 341 significantly reduced by $35 \%$ and $18.5 \%$, respectively compared to control cells in 342 response to $\mathrm{RNase} \mathrm{H}$ treatment (Fig. 4c, d). Finally, we also depleted R-loops in vivo 343 by overexpressing ppyCAG-v5-RNaseH1 in cells. IF revealed that nuclear S9.6 levels 344 were significantly reduced in cells which expressed v5 (to $38 \%$ of controls) and that mean levels of chromatin-associated SA1 were similarly reduced by $29.4 \%$ ( $p=4.05 E-$ 8) (Fig.S4c), further confirming the causal relationship between R-loops and SA 347 proteins.

\section{SA1 proteins act as suppressors of R-loops.}

350 Proteins that act to suppress R-loops in vivo, such as $A Q R{ }^{36}$, have an inverse correlation with S9.6 levels. From our IF results (Fig. 4c, d), we noticed that SA1 had a similar negative relationship with S9.6 (Fig. S4d), prompting us to investigate whether SA proteins could act as suppressors of R-loop formation. To this end, we treated RAD21 ${ }^{\mathrm{mAC}}$ cells with scramble control siRNAs or siRNA to SA1, SA2 or AQR and used IF to assess the impact on nuclear S9.6 levels in KD cells (Fig.4e, f). As previously reported, AQR KD resulted in a $30.1 \%$ increase of mean nuclear 59.6 levels $(p=0.0004)$. Compared to control siRNA-treated cells, mean SA1 levels were reduced by $56.2 \%(p=4.1 E-40)$, while mean nuclear 59.6 staining was significantly increased in the same cells by $55.3 \%(p=3.90 E-08)$ (Fig.3f, $g)$. We note that perturbing SA1 levels increased nuclear S9.6 staining to a similar extent as what was observed upon AQR KD, a bonafide R-loop regulator. When we treated cells with the custom siRNA to SA1 e31 (Fig 3e), we also observed an increase in S9.6 signals (Fig S4e), suggesting that this basic exon plays a role in R-loop stability. Surprisingly, despite efficient KD of SA2 (68\% reduction), there was no significant change in nuclear S9.6 staining (mean $\mathrm{S} 9.6$ reduced by $10 \%$ compared to control, $\mathrm{p}=0.17$ ), indicating that although SA2 is localised to R-loops (Fig S4b), it does not seem to contribute to their regulation. Taken together, our results confirm the presence of SA proteins at endogenous R-loops in vivo and reveal a role for SA1 in R-loop suppresion. 
370 SA contributes to cohesin loading independently of NIPBL.

371 Our results thus far support a hypothesis whereby $\mathrm{SA}^{\Delta \mathrm{Coh}}$ engages with RNA and

372 various RNA binding proteins at clustered regulatory regions (possibly R-loops) to 373 structurally support them and/or facilitate cohesin's association with chromatin.

374 Indeed, several lines of evidence suggest that alongside the canonical NIPBL/Mau2

375 loading complex, SA proteins contribute to cohesin's association with chromatin. In

376 yeast, interaction of the SA orthologue with the loader complex is required for efficient

377 association of the cohesin ring with DNA and subsequent ATPase activation ${ }^{13,14}$.

378 Separating interactions into SA-loader and cohesin ring-loader subcomplexes still

379 impairs cohesin loading, indicating that SA functions as more than just a bridge protein

$380{ }^{14}$. Crystallisation studies reveal a striking similarity of NIPBL and SA, in that both are

381 highly bent, HEAT-repeat proteins ${ }^{39,40}$. Indeed, NIPBL and SA1 interact together in

382 an antiparallel arrangement and wrap around DNA and the cohesin ring via similar

383 interactions in their respective ' $U$ ' surfaces, implying that SA1 has a role in the initial

384 recruitment of cohesin to DNA alongside NIPBL (Shi 2020, Higashi 2020).

385 The RAD21 ${ }^{\mathrm{mAC}}$ system has the unique advantage that when IAA is washed off

386 cells, RAD21 proteins are no longer degraded and can be 're-loaded' back onto

387 chromatin (Fig.5a). We coupled this to an siRNA-mediated KD of NIPBL to investigate

388 whether cohesin re-loading onto chromatin is influenced by SA proteins in human cells

389 in native conditions. RAD2 $1^{\mathrm{mAC}}$ cells were treated with scramble or NIPBL siRNAs and

390 subsequently grown in ethanol or IAA. The 'Oh post IAA wash-off' sample represents

391 the extent of cohesin degradation in the IAA-treated cells. In parallel, IAA was washed

392 out and the cells were left for $4 \mathrm{~h}$ to recover. This sample, ' $4 \mathrm{~h}$ post IAA wash-off'

393 represents the extent of cohesin re-loading in the respective genetic background

394 (Fig.5a, b). We confirmed loss of the loader complex by immunoblot for both NIPBL

395 and MAU2 as it is known that MAU2 is de-stablised upon NIPBL loss ${ }^{41}$. We note that

396 re-loading was not fully restored to the levels observed in ethanol-treated cells and

397 varied between experiments (Fig.5c), which may reflect differences in the initial

398 amounts of RAD21 or NIPBL (see methods). Despite this variation, we observed a

399 consistent effect on RAD21 re-loading across 8 independent experiments. As

400 expected, in NIPBL KD conditions, mean RAD21 re-loading efficiency was reduced to

$40140.9 \%$ of the siRNA controls (mean re-loading siNIPBL, 2.1 vs siCon, 3.6), however 
402

403

404

405

406

407

408

409

410

411

412

413

414

415

416

417

418

419

420

421

422

423

424

425

426

427

428

429

430

this did not represent a statistically significant difference $(p=0.33)$ and accordingly, a large fraction of chromatin-associated RAD21 could still be detected in NIPBL KD cells (Fig. 5c), indicating that cells have a NIPBL-independent cohesin re-loading mechanism.

We performed the same experiment and this time, in addition to treating cells with siRNAs to NIPBL, we also included siRNA to SA1 and SA2 together (siSA), and a siNIPBL+ siSA condition to ask if SA proteins contribute to the observed NIPBLindependent reloading. Across 5 independent experiments, SA KD had a more dramatic effect on cohesin re-loading efficiency than NIPBL KD, reducing RAD21 reloading on chromatin to $51 \%$ of scramble controls (mean re-loading siSA, 1.9 vs siCon, 5.1, p=0.061), (Fig. 5d, e). However, only when SA and NIPBL were both reduced in cells, was there a statistically significant change to cohesin re-loading, reducing RAD21 on chromatin to $64.9 \%$ of scramble control cells (mean re-loading siNIPBL+siSA, 1.42 vs siCon, $5.1, p=0.001$ ), indicating that $S A$ performs an important and complementary step to NIPBL and MAU2 during normal reloading (Fig.5d, e).

Finally, given that SA localises to R-loops and these can be localized to many places across the genome, we reasoned that SA could use this structural platform to link the loading of cohesin to diverse biological processes. Therefore, we repeated the cohesin re-loading experiments in the presence of siRNAs to AQR, which we had previously shown act as suppressors of R-loops (Fig. 4e, f). AQR KD alone had little effect on cohesin re-loading efficiency (Fig. $5 f, g$ ), however when R-loops were increased in the context of reduced NIPBL, we observed an increase in the efficiency of cohesin re-loading compared to control cells (Fig. $5 f, g$ ). This increase in re-loading efficiency corresponded with a 2.08-fold increase in SA1 levels and a 1.46-fold increase in s9.6 levels, relative to siCon (Fig. S5a) while MAU2 and AQR showed a corresponding fold-change of 0.48 and 0.69 , respectively, indicating the specificity of SA1 and R-loop increase. Our results support a role for R-loops in SA-mediated cohesin loading. 


\section{DISCUSSION}

432 Whether SA proteins function in their own right outside of the cohesin complex is rarely

433 considered. Consequently, our understanding of how these proteins contribute to

434 cohesin function and disease is incomplete. In this study, we shed light on this

435 question by uncovering a diverse repertoire of SA interactors in cells acutely depleted

436 for the cohesin trimer. These range from proteins associated with translation and

437 ribogenesis to RNA processing factors and regulators of the epitranscriptome. These

438 observations suggest that SA proteins have a previously unappreciated role in post-

439 transcriptional regulation of gene expression which offers much-needed new insight

440 into its roles in disease and cancer.

Acute depletion of the cohesin ring has allowed us to capture a moment in the

442 normal life cycle of cohesin - DNA associations and unveiled a previously

443 unappreciated step for SA proteins. We show that cohesin-independent SA proteins

444 bind to DNA and RNA, in the context of non-canonical RNA:DNA hybrid structures as

445 we have shown here, or sequentially, and use this platform for the loading of cohesin

446 to chromatin. Our results are supportive of biophysical observations of SA proteins

447 and R-loops ${ }^{42}$ and in vitro assessment of cohesin loading at DNA intermediates ${ }^{24}$.

448 Structural studies suggest that NIPBL and SA1 together bend DNA and cohesin to

449 guide DNA entering into the cohesin ring ${ }^{17,18,43}$. Our work shows that in cells lacking

450 either the canonical NIPBL/MAU2 loader complex or the SA proteins, cohesin can still

451 associate with chromatin, suggesting that loading can occur with either component

452 alone, albeit most effectively together.

453 Since SA paralogues have distinct terminal ends and nucleic acid targeting 454 mechanisms ${ }^{22,23}$, their initial recruitment to chromatin may be specified by unique 455 DNA, RNA or protein-interactions, or indeed all three. Such diversification of loading 456 platforms would be important in large mammalian genomes to ensure sufficient 457 cohesin was chromatin associated or to direct stabilization of particular biological 458 processes for a given cell fate ${ }^{44}$. Indeed, SA1 and SA2 show clear differences in 459 interaction with FGF-motif containing proteins, despite the fact that both paralogs 460 contain a CES domain ${ }^{45}$, underscoring the importance of in vivo studies and arguing 461 that additional factors play an important role in complex stabilization. In this context, 462 RNA-associated protein interaction has previously been shown to support cohesin 
463 stabilisation at CTCF at the IGF2/H19 locus ${ }^{46}$. These results are in line with our

464 findings that a basic domain in the unstructured C-terminal portion of SA supports

465 RNA-associated protein interactions and R-loop stability.

466 This study identifies SA proteins as novel regulators of RNA:DNA hybrid

467 homeostasis. It is noteworthy that other suppressors of R-loop formation include

468 mRNA processing factors, chromatin remodellers and DNA repair proteins ${ }^{47}$ which all

469 function in the context of nuclear bodies ${ }^{48}$. We find that SA proteins are enriched at

470 very distal chromatin interactions in cohesin-depleted $\mathrm{Hi}-\mathrm{C}$ data and they interact with

471 numerous RNA binding proteins known to condense in 3D ${ }^{49,50}$. Harnessing such

472 condensates would provide an efficient loading platform for cohesin at sites of similar

473 biological function. If SA paralogs direct different localization of cohesin loading or

474 stability of its association, this could have important implications in our understanding

475 of disease and cancer. 


\section{ACKNOWLEDGMENTS}

477 This work would not be possible without the support of a Senior Research Fellowship 478 from the Wellcome Trust awarded to S.H. (106985/Z/15/Z) and a CRUK PhD 479 studentship awarded to H.P. The Proteomics work was supported by the CRUK-UCL 480 Centre Award [C416/A25145]. We thank Stanimir Dulev for his contributions at the 481 early stages of the project. We would like to thank Konstantina Skourti-Stathaki for 482 advice about S9.6 IFs and R-loops. We are grateful to the members of the Hadjur lab 483 for critical discussions and reading of the manuscript.

\section{AUTHOR CONTRIBUTIONS}

486 H.P. and S.H. conceived the project. H.P. designed and performed all the colP, Mass spectrometry and cohesin re-loading experiments, analysed the ChIP and $\mathrm{Hi}-\mathrm{C}$ data and performed the statistical analysis for mass spectrometry with the support of A.B. and S.S. Y.L. performed and analysed all imaging experiments (apart from STORM), derived clonal lines of RAD21-mAC cells, cloned YFP-tagged SA2 cDNAs and performed CLIP together with M.T.C. W.V. performed Hi-C, ChIP-seq and splicing analyses. M.V.N., L.M. and M.P.C. performed and analysed STORM imaging. D.P. discovered splicing features of the SA isoforms. H.P. and Y.L. prepared cellular

494 materials for CLIP, which was carried out by M.B., M.T.C., and R.J. A.B. and S.S. 495 performed mass spectrometric and proteomic analysis. H.P., Y.L and S.H. formatted 496 all figures and wrote the manuscript with input from all authors.

\section{Declaration of Interests}

499 The authors declare no competing interests. 


\section{FIGURE LEGENDS}

Figure 1. SA interacts with CTCF in the absence of cohesin.

A) Representative confocal images of SA1 and CTCF IF in RAD21 ${ }^{\mathrm{mAC}}$ cells treated with ethanol $(\mathrm{EtOH})$ as a control or Auxin (IAA) for $4 \mathrm{hrs}$. Nuclei were counterstained with DAPI.

B) Imaris quantification of the fluorescence intensity of mClover, SA1 (top panel), SA2 (bottom panel) and CTCF in EtOH and IAA-treated RAD21 ${ }^{\mathrm{mAC}}$ cells. Whiskers and boxes indicate all and $50 \%$ of values respectively. Central line represents the median. Asterisks indicate a statistically significant difference as assessed using two-tailed ttest. ${ }^{\star} p<0.05,{ }^{* *} p<0.005,{ }^{* * *} p<0.0005$, n.s., not significant. $n>50$ cells/condition from 3 biological replicates.

Chromatin colP of (C) SA1, SA2 and IgG with RAD21 and CTCF or (D) CTCF and IgG with RAD21, SA1 and SA2 in RAD21 ${ }^{\mathrm{mAC}}$ cells treated with EtOH or IAA for 4hrs. Input represents (C) $2.5 \%$ and (D) $1.25 \%$ of the material used for immunoprecipitation.

E) Dual-color STORM images of SA1 (green) and CTCF (magenta) in EtOH and IAAtreated RAD2 $1^{\mathrm{mAC}}$ cells. Representative full nuclei and zoomed nuclear areas are shown. Line denotes 2 microns and 200nm for full nuclei and zoomed areas respectively. See supplementary Figures for SA2 STORM images.

F) Mean CTCF, SA1 and SA2 localization densities (localizations normalized with nuclear area) in EtOH and IAA-treated RAD2 $1^{\mathrm{mAC}}$ cells $(\mathrm{n}=>30,>17$ and $>15$ nuclei for CTCF, SA1 and SA2 respectively). Mean and SD are plotted, Mann Whitney test. $\star * \star * \quad p<0.0001$.

G) Mean Nearest Neighbor Distance (NND) of CTCF, SA1 and SA2 clusters in nanometers in $\mathrm{EtOH}$ and IAA-treated cells $(n=>38,>14$ and $>23$ nuclei for CTCF, SA1 and SA2 respectively). Mean and SD are plotted, Mann Whitney test. ${ }^{* \star * *}$ $\mathrm{p}<0.0001$.

H) Nearest Neighbor Distance (NND) distribution plot of the distance between CTCF and SA1 (left panel) or SA2 (right panel) clusters in EtOH and IAA-treated cells. Experimental data are shown as continuous lines, random simulated data are displayed as dotted lines.

I) ChIP-seq heat map of CTCF, SA1, SA2, Rad21 and 540 SMC3 binding profiles in control (EtOH) and IAA-treated RAD21 ${ }^{\mathrm{mAC}}$ cells. Selected regions are bound by CTCF in control conditions.

J) Analysis of contact frequency hotspots from Hi-C libraries generated from EtOHtreated (top row) and IAA-treated (bottom row) RAD2 ${ }^{\mathrm{mAC}}$ cells. Contact frequencies were calculated in two distance ranges of $100 \mathrm{~kb}-1 \mathrm{Mb}$ and $1-5 \mathrm{Mb}$. The last column includes contact frequencies specifically at residual SA-CTCF binding sites. 
Figure 2. Characterization of SA1 protein-protein interaction network in RAD21depleted cells.

A) Chromatin colP of SA1, SA2 and IgG with 4 predicted CES-binding proteins in RAD2 $1^{\mathrm{mAC}}$ cells treated with $\mathrm{EtOH}$ or IAA for $4 \mathrm{hrs}$. Input represents $1.25 \%$ of the material used for immunoprecipitation.

553

B) Volcano plot displaying the statistical significance (-log10 p-value) versus magnitude of change (log2 fold change) from SA1 IP-MS data produced from RAD $21^{\mathrm{mAC}}$ cells treated with ethanol or IAA $(n=3)$. Vertical dashed lines represent changes of 1.5-fold. Horizontal dashed line represents a pvalue of 0.1 . Cohesin complex members and validated high-confidence proteins have been highlighted.

C) $S A 1^{\Delta C o h}$ interaction network of protein-protein interactions identified in RAD21 ${ }^{\mathrm{mAC}}$ cells using STRING. Node colours describe the major enriched categories, with squares denoting helicases and polygons denoting hnRNP family members. Proteins within each enrichment category were subset based on p-value change in B). See supplemental figures for full network.

D) Chromatin IP of SA1 and IgG in RAD21 ${ }^{\mathrm{mAC}}$ cells treated with $\mathrm{EtOH}$ or IAA and immunoblotted with antibodies to validate the proteins identified by IP-MS. Input represents $1.25 \%$ of the material used for immunoprecipitation. ${ }^{*}$ We note that ESYT2 is a FGF-containing protein.

Figure 3. SA proteins bind to RNA.

A) CLIP for SA1, SA2 and non-specific IgG controls. Autoradiograms of crosslinked ${ }^{32} \mathrm{P}$-labelled RNA are shown at the top and the corresponding immunoblots, below. CLIP was performed with and without UV crosslinking and polynucleotide kinase (PNK) and with high $(\mathrm{H} ; 1 / 50$ dilution) or low ( $\mathrm{L} ; 1 / 500$ dilution) concentrations of RNase I.

B) CLIP for SA1, SA2 and non-specific IgG control in ethanol (-) or IAA-treated Rad $21^{\mathrm{mAC}}$ cells. ${ }^{32} \mathrm{P}$-labelled RNA and the corresponding immunoblots are shown as above.

C) Schematic of the SA1 and SA2 proteins showing the SA1-specific AT-hook, the conserved CES domain (blue) and the acidic C-terminus (green) which contains the basic alternatively spliced exon (red). Right-hand zoom-in indicates the spliced exons for SA1 (top) and SA2 (bottom) and the pl for each. The conservation scores for the divergent $\mathrm{N}$ - and $\mathrm{C}$-termini and the middle portion of the proteins which contains the CES domain are shown.

(D) Percent Spliced In (PSI) calculations for SA1 exon 31 (black) and SA2 exon 32 (grey) based on VAST-Tools analysis of RNA-seq from multiple datasets (see Methods). 
(E) Western blot analysis of SA1 levels in whole cell lysates after treatment with scrambled siRNAs (siCon), SmartPool SA1 siRNAs (siSA1 SP), control esiRNAs (esiCon) and esiRNA designed to target SA1 exon 31 for $48 \mathrm{hrs}$ in RAD2 $1^{\mathrm{mAC}}$ cells. H3 serves as a loading control. The percentage of knockdown (KD) after $\mathrm{SA} 1$ signal is normalised to $\mathrm{H} 3$ is shown.

(F) Western blot analysis of SA2 levels in whole cell lysates after treatment with scrambled siRNAs (siCon), SmartPool SA2 siRNAs (siSA2 SP), control esiRNAs (esiCon) and siRNA designed to target SA2 exon 32 for $48 \mathrm{hrs}$ in RAD2 ${ }^{\mathrm{mAC}}$ cells. H3 serves as a loading control. . The percentage of knockdown (KD) after $\mathrm{SA} 2$ signal is normalised to $\mathrm{H} 3$ is shown.

(G) CLIP with endogenous SA2 (as in A, B above), IgG control and cells where either YFP-tagged full-length SA2 or YFP-tagged SA2 lacking exon 32 are expressed for $48 \mathrm{hrs}$. CLIP reveals proteins which specifically associate with exon-32 containing SA2 (arrow).

\section{Figure 4. SA proteins act as suppressors of endogenous R-loops.}

(A) Adjusted p-value (FDR) for enrichment of S9.6 interactome data from Cristini et al. and Wang et al., with the $S A 1^{\triangle \mathrm{Coh}}$ interactome. Overlap indicates the proteins identified in both S9.6 interactome datasets, representing a high confidence R-loop interactome list.

(B) Chromatin colP of S9.6 and IgG in RAD2 $1^{\mathrm{mAC}}$ cells treated with RNase $\mathrm{H}$ enzyme and immunoblotted with antibodies representing known R-loop proteins, as well as SA1. Input represents $1.25 \%$ of the material used for immunoprecipitation. Bottom, S9.6 dot blot of lysates used in colP.

(C) Representative confocal images of S9.6, SA1 and SA2 IF in RAD21 ${ }^{\text {mAC }}$ cells treated with control buffer or RNase $\mathrm{H}$ enzyme. Nuclear outlines (white) are derived from DAPI counterstain.

(D) Fluorescence Intensity of S9.6, SA1 and SA2 protein assessed by Immunofluorescence in (C). Data are from three biological replicates with $>50$ cells counted/condition). Quantifications and statistical analysis were done as above.

(E) Representative confocal images of $\mathrm{S} 9.6$ and SA1, SA2 or AQR IF in RAD21 ${ }^{\mathrm{mAC}}$ cells treated with scramble control siRNA (si scr) or siRNA to the protein of interest. Nuclear outlines (white) are derived from DAPI counterstain.

(F) Fluorescence Intensity of S9.6, SA1, SA2 and AQR proteins assessed by Immunofluorescence in (E). Data are from three biological replicates with $>50$ cells counted/condition). Quantifications and statistical analysis were done as previously stated. 
644 Figure 5. SA proteins contribute to cohesin loading at R-loops.

645 (A) Schematic of experimental set-up. RAD21 $1^{\mathrm{mAC}}$ cells expressing mClover (green cells in dishes) were treated with scramble siRNAs or siRNA to NIPBL. Prior to collection, cells were cultured in ethanol or IAA for $4 \mathrm{hrs}$ to degrade RAD21 $(0 \mathrm{~h}$ timepoints). Each sample was then split into those cultured in ethanol or IAA for 4hrs to degrade RAD21 (0h timepoints). The IAA treatment was washed-off and the cells were left to recover for $4 \mathrm{hrs}$ ( $4 \mathrm{~h}$ timepoints). Chromatin fractions were prepared from all samples and used in immunoblot analysis.

(B) Representative western blot analysis of chromatin-bound RAD21, MAU2 and NIPBL levels in RAD2 $1^{\mathrm{mAC}}$ cells treated according to the schematic shown in (A). H3 was used as a loading control. NB The full blots can be seen in the supplement.

(C) RAD21 fold change relative to siCon samples at the $0 \mathrm{~h}$ timepoint in siCon $4 \mathrm{~h}$ (light grey), siNIPBL Ohr (light blue) and siNIPBL 4hr (dark blue). Whiskers and boxes indicate all and $50 \%$ of values respectively. Central line represents the median. statistical analysis as assessed using a two-tailed t-test. Data is from 8 biological replicates.

(D) Representative western blot analysis of chromatin-bound RAD21, SA1, SA2, MAU2 and NIPBL levels in RAD21mAC cells treated according to the schematic shown in (A) and including samples treated with siRNA to SA1 and SA2 together (siSA) and siRNA to NIPBL + siSA. H3 was used as a loading control.

(E) RAD21 fold change relative to siCon samples at the $0 \mathrm{~h}$ timepoint in siCon, siNIPBL, siSA and siNIPBL+siSA. Asterisks indicate a statistically significant difference as assessed using 2-tailed T-test. ${ }^{* *} \mathrm{p}<0.005$. Data is from 5 biological replicates.

(F) Representative western blot analysis of chromatin-bound RAD21, AQR, MAU2 and NIPBL levels in RAD21 mAC cells treated according to the schematic shown in $(A)$ and including samples treated with siRNA to AQR and siRNA to NIPBL + AQR. H3 was used as a loading control. RAD21* denotes increased exposure. NIPBL levels could not be determined due to incompatibility with assessing RNA:DNA hybrids. NB siCon samples were cropped here and the full blots can be seen in the supplement.

(G) RAD21 fold change relative to siCon samples at the Oh timepoint in siCon, siNIPBL, siAQR and siNIPBL+siAQR. Data is from 2 biological replicates and is represented as mean +/- SEM. 


\section{REFERENCES}

6871.

688

689

690

691

692

693

694

695

696

697

698

699

700

701

702

703

704

705

706

707

708

709

710

711

712

713

714

715

716

717

718

719

720

721

722

723

724

725

726

727

728

729

730

731

732

733

734

735

736

737

738

739

740

741

Leiserson, M. D. M. et al. Pan-cancer network analysis identifies combinations of rare somatic mutations across pathways and protein complexes. Nat. Genet. 47, 106-114 (2015).

2. Horsfield, J. A. et al. Cohesin-dependent regulation of Runx genes. Development 134, 2639-2649 (2007).

3. Viny, A. D. \& Levine, R. L. Cohesin mutations in myeloid malignancies made simple. Curr. Opin. Hematol. 25, 61-66 (2018).

4. Hadjur, S. et al. Cohesins form chromosomal cis-interactions at the developmentally regulated IFNG locus. Nature 460, 410-413 (2009).

5. Sofueva, S. et al. Cohesin-mediated interactions organize chromosomal domain architecture. EMBO J. 32, 3119-3129 (2013).

6. Zuin, J. et al. Cohesin and CTCF differentially affect chromatin architecture and gene expression in human cells. Proc. Natl. Acad. Sci. U.S.A. 111, 996-1001 (2014).

7. Kojic, A. et al. Distinct roles of cohesin-SA1 and cohesin-SA2 in 3D chromosome organization. Nature Publishing Group 25, 496-504 (2018).

8. Rao, S. S. P. et al. Cohesin Loss Eliminates All Loop Domains. Cell 171, 305-309.e24 (2017).

9. Wutz, G. et al. Topologically associating domains and chromatin loops depend on cohesin and are regulated by CTCF, WAPL, and PDS5 proteins. EMBO J. 36, 35733599 (2017).

10. Fudenberg, G., Abdennur, N., Imakaev, M., Goloborodko, A. \& Mirny, L. Emerging Evidence of Chromosome Folding by Loop Extrusion. bioRxiv 1-24 (2018). doi:10.1101/264648

11. Balbás-Martínez, C. et al. Recurrent inactivation of STAG2 in bladder cancer is not associated with aneuploidy. Nat. Genet. 45, 1464-1469 (2013).

12. Solomon, D. A. et al. Frequent truncating mutations of STAG2 in bladder cancer. Nat. Genet. 45, 1428-1430 (2013).

13. Murayama, Y. \& UhImann, F. Biochemical reconstitution of topological DNA binding by the cohesin ring. Nature 505, 367-371 (2014).

14. Orgil, O. et al. A conserved domain in the scc3 subunit of cohesin mediates the interaction with both mcd1 and the cohesin loader complex. PLoS Genet. 11, e1005036 (2015).

15. Ciosk, R. et al. Cohesin's Binding to Chromosomes Depends on a Separate Complex Consisting of Scc2 and Scc4 Proteins. Molecular Cell 5, 243-254 (2000).

16. Li, Y. et al. Structural basis for Scc3-dependent cohesin recruitment to chromatin. eLIFE 7, 352 (2018).

17. Shi, Z., Gao, H., Bai, X.-C. \& Yu, H. Cryo-EM structure of the human cohesin-NIPBLDNA complex. Science 368, 1454-1459 (2020).

18. Higashi, T. L. et al. A Structure-Based Mechanism for DNA Entry into the Cohesin Ring. Molecular Cell 79, 917-933.e9 (2020).

19. Xiao, T., Wallace, J. \& Felsenfeld, G. Specific sites in the $C$ terminus of CTCF interact with the SA2 subunit of the cohesin complex and are required for cohesin-dependent insulation activity. Mol. Cell. Biol. 31, 2174-2183 (2011).

20. Saldaña-Meyer, R. et al. CTCF regulates the human p53 gene through direct interaction with its natural antisense transcript, Wrap53. Genes Dev. 28, 723-734 (2014). Bisht, K. K., Daniloski, Z. \& Smith, S. SA1 binds directly to DNA through its unique AThook to promote sister chromatid cohesion at telomeres. J. Cell. Sci. 126, 3493-3503 (2013).

22. Lin, J. et al. Functional interplay between SA1 and TRF1 in telomeric DNA binding and DNA-DNA pairing. Nucleic Acids Research 44, 6363-6376 (2016).

23. Countryman, $\mathrm{P}$. et al. Cohesin SA2 is a sequence-independent DNA-binding protein that recognizes DNA replication and repair intermediates. J. Biol. Chem. 293, 1054-1069 (2018).

24. Murayama, Y., Samora, C. P., Kurokawa, Y., Iwasaki, H. \& Uhlmann, F. Establishment of DNA-DNA Interactions by the Cohesin Ring. Cell 1-29 (2018). 
bioRxiv preprint doi: https://doi.org/10.1101/2021.02.20.432055; this version posted February 21, 2021. The copyright holder for this preprint (which was not certified by peer review) is the author/funder, who has granted bioRxiv a license to display the preprint in perpetuity. It is made available under aCC-BY 4.0 International license.

doi:10.1016/j.cell.2017.12.021

25. Zheng, G., Kanchwala, M. \& Xing, C. MCM2-7-dependent cohesin loading during $S$ phase promotes sister-chromatid cohesion. eLIFE 1-25 (2018). doi:10.7554/eLife.33920.001

26. Natsume, T., Kiyomitsu, T., Saga, Y. \& Kanemaki, M. T. Rapid Protein Depletion in Human Cells by Auxin-Inducible Degron Tagging with Short Homology Donors. Cell Rep 15, 210-218 (2016).

27. Parelho, V. et al. Cohesins functionally associate with CTCF on mammalian chromosome arms. Cell 132, 422-433 (2008).

28. Wendt, K. S. et al. Cohesin mediates transcriptional insulation by CCCTC-binding factor. Nature 451, 796-801 (2008).

29. Roig, M. B. et al. Structure and function of cohesinâ $€^{\mathrm{TM}} \mathrm{S} \operatorname{Scc} 3 / \mathrm{SA}$ regulatory subunit. FEBS Letters 588, 3692-3702 (2014).

30. Hons, M. T. et al. Topology and structure of an engineered human cohesin complex bound to Pds5B. Nature Communications 7, 1-11 (2017).

31. Kim, J.-S. et al. Systematic proteomics of endogenous human cohesin reveals an interaction with diverse splicing factors and RNA-binding proteins required for mitotic progression. J. Biol. Chem. 294, 8760-8772 (2019).

32. Irimia, M. et al. A Highly Conserved Program of Neuronal Microexons Is Misregulated in Autistic Brains. Cell 159, 1511-1523 (2014).

33. Santos-Pereira, J. M. \& Aguilera, A. R loops: new modulators of genome dynamics and function. Nat. Rev. Genet. 16, 583-597 (2015).

34. Cristini, A., Groh, M., Kristiansen, M. S. \& Gromak, N. RNA/DNA Hybrid Interactome Identifies DXH9 as a Molecular Player in Transcriptional Termination and R-LoopAssociated DNA Damage. Cell Rep 23, 1891-1905 (2018).

35. Wang, I. X. et al. Human proteins that interact with RNA/DNA hybrids. Genome Res. 28, 1405-1414 (2018).

36. Sollier, J. et al. Transcription-coupled nucleotide excision repair factors promote R-loopinduced genome instability. Molecular Cell 56, 777-785 (2014).

37. Skourti-Stathaki, K., Kamieniarz-Gdula, K. \& Proudfoot, N. J. R-loops induce repressive chromatin marks over mammalian gene terminators. Nature 516, 436-439 (2014).

38. Cerritelli, S. M. \& Crouch, R. J. Ribonuclease H: the enzymes in eukaryotes. FEBS J. 276, 1494-1505 (2009).

39. Kikuchi, S., Borek, D. M., Otwinowski, Z., Tomchick, D. R. \& Yu, H. Crystal structure of the cohesin loader Scc2 and insight into cohesinopathy. Proc. Natl. Acad. Sci. U.S.A. 113, 12444-12449 (2016).

40. Hara, K. et al. Structure of cohesin subcomplex pinpoints direct shugoshin-Wapl antagonism in centromeric cohesion. Nature Publishing Group 21, 864-870 (2014).

41. Watrin, E. et al. Human Scc4 is required for cohesin binding to chromatin, sisterchromatid cohesion, and mitotic progression. Curr. Biol. 16, 863-874 (2006). Pan, $\mathrm{H}$. et al. Cohesin SA1 and SA2 are RNA binding proteins that localize to RNA containing regions on DNA. Nucleic Acids Research 24, 105-17 (2020).

43. Chao, W. C. H. et al. Structural Studies Reveal the Functional Modularity of the Scc2Scc4 Cohesin Loader. Cell Rep 12, 719-725 (2015).

44. Pezic, D. et al. The cohesin regulator Stag1 promotes cell plasticity through heterochromatin regulation. bioRxiv 1-60 (2021). doi:10.1101/2021.02.14.429938

45. $\quad \mathrm{Li}, \mathrm{Y}$. et al. The structural basis for cohesin-CTCF-anchored loops. Nature $\mathbf{5 7 8 , 4 7 2 - 4 7 6}$ (2020).

46. Yao, H. et al. Mediation of CTCF transcriptional insulation by DEAD-box RNA-binding protein p68 and steroid receptor RNA activator SRA. Genes Dev. 24, 2543-2555 (2010).

47. García-Muse, T. \& Aguilera, A. R Loops: From Physiological to Pathological Roles. Cell 1-15 (2019). doi:10.1016/j.cell.2019.08.055

48. Misteli, T. Beyond the Sequence: Cellular Organization of Genome Function. Cell 128, 787-800 (2007).

49. Nozawa, R.-S. et al. SAF-A Regulates Interphase Chromosome Structure through Oligomerization with Chromatin- Associated RNAs. Cell 169, 1214-1227.e18 (2017). Huo, X. et al. The Nuclear Matrix Protein SAFB Cooperates with Major Satellite RNAs to 
bioRxiv preprint doi: https://doi org/10.1101/2021.02 20.432055; this version posted February 21, 2021. The copyright holder for this preprint (which was not certified by peer review) is the author/funder, who has granted bioRxiv a license to display the preprint in perpetuity. It is made available under aCC-BY 4.0 International license.

Stabilize Heterochromatin Architecture Partially through Phase Separation. Molecular Cell 77, 368-383.e7 (2020).

51. Barrington, C., Georgopoulou, D., Nature, D. P.2019. Enhancer accessibility and CTCF occupancy underlie asymmetric TAD architecture and cell type specific genome topology. Nat Communications. doi:10.1038/s41467-019-10725-9

52. Cox, J. \& Mann, M. MaxQuant enables high peptide identification rates, individualized p.p.b.-range mass accuracies and proteome-wide protein quantification. Nat Biotechnol

53. Choi, M. et al. MSstats: an R package for statistical analysis of quantitative mass spectrometry-based proteomic experiments. Bioinformatics 30, 2524-2526 (2014).

54. Beltran, M. et al. The interaction of PRC2 with RNA or chromatin is mutually antagonistic. Genome Res. 26, 896-907 (2016). 


\section{METHODS}

814 Cell culture and IAA-mediated degradation of Rad21.

815 HCT116 cells with engineered RAD21-miniAID-mClover (RAD21mAC), or OsTIR1-

816 only, or both (RAD21mAC-OsTIR) were obtained from Masato T. Kanemaki. The cells 817 were maintained in McCoy's 5A medium with Glutamax (Thermo Fisher Scientific) 818 supplemented with $\quad 10 \%$ Heat-inactivated FBS 819 (Gibco), $700 \mu \mathrm{g} / \mathrm{ml}$ Geneticin, $100 \mu \mathrm{g} / \mathrm{ml}$ Hygromycin B Gold and 100 $\mu \mathrm{g} / \mathrm{ml}$ Puromycin 820 as described in (Natsume). We clonally selected the RAD21mAC-OsTIR cells by 821 sorting green fluorescence positive single cells on a FACS Aria Fusion cell sorter (BD 822 Bioscience). Single cells were individually seeded into one well of a 96-well plate, 823 expanded for 10 days into $6 \mathrm{~cm}$ culture dishes and selected with Geneticin, 824 Hygromycin B Gold and Puromycin as indicated above in McCoy's medium for another 82510 days. Each clone was assessed for efficiency of Rad21 degradation using FACS 826 analysis and western blotting (WB) using mClover, mAID and OsTIR antibodies. Two 827 clones ( $\mathrm{H} 2$ and $\mathrm{H} 11)$ were taken forward and used throughout this study. To deplete 828 RAD21, RAD21mAC-OsTIR cells were grown in adherent conditions for 3 days and 829 treated with $500 \mu \mathrm{M}$ Indole-3-acetic acid (IAA, Auxin, diluted in ethanol) for 4 830 hours. For IAA withdrawal, IAA treated cells were washed with PBS and replaced with 831 fresh supplemented McCoy's medium for another 4 hours. Cells were washed twice with ice-cold PBS before being harvested for later experimental procedures.

\section{siRNA-mediated knockdowns.}

835 For siRNA transfections, RAD21 ${ }^{\mathrm{mAC}}$-OsTIR cells were reverse transfected with 836 scramble siRNA (siCon) or siRNAs targeting SA1, SA2, NIPBL, or AQR (Dharmacon, 837 Horizon Discovery). A final concentration of $10 \mathrm{nM}$ of siSA1, siSA2, or siNIPBL or $8385 \mathrm{nM}$ of siAQR was reverse transfected into the cells using 839 Lipofectamine RNAiMAX reagent (Invitrogen), as per the manufacturer's 840 instructions. Cells were plated at a density of $1-1.25 \times 10^{6}$ cells per $10 \mathrm{~cm}$ dish and 841 harvested $72 \mathrm{hrs}$ post-transfection, at a confluency of $\sim 70 \%$. The Lipofectamine842 containing media was replaced with fresh media 12-16 hrs post-transfection to avoid 843 toxicity. For Figure 5f/g, incubation time was reduced to $48 \mathrm{hrs}$. To account for the 844 reduced growth time, cells were plated at a density of $2-3 \times 10^{6}$ cells per $10 \mathrm{~cm}$ dish. 845 Here siCon- and siNIPBL-transfected cells were plated at a lower cell number 846 than siAQR-transfected cells to ensure equalised confluence $(\sim 70 \%)$ at the time of 847 collection. When IAA-treatment was combined with siRNA mediated KD, the IAA was 848 added at the end of the normal KD condition so that total KD time was not changed 849 compared to UT cells. For esiRNA treatment, RAD21 ${ }^{\mathrm{mAC}}$-OsTIR cells were reverse850 transfected with $20 \mu \mathrm{M}$ FLUC control esiRNA or esiRNA custom designed to SA1 851 exon31 or SA2 exon32 (MISSION® SiRNA, Sigma Aldrich) 852 using RNAiMAX (Invitrogen). Cells were incubated in transfection mixture for 7-8 853 hours before being replaced with fresh supplemented McCoy's medium and left for 
854

855

856

857

858

859

860

861

862

863

864

865

866

867

868

869

870

871

872

873

874

875

876

877

878

879

880

881

882

883

884

885

886

887

888

889

890

891

892

893

894

another 40h until harvest. Efficiency of KD was assessed by WB. siRNA information can be found in Table 1.

\section{Immunofluorescence}

Cells were adhered onto poly L-lysine coated glass coverslips in 6 well culture dishes and were washed twice with ice-cold PBS before IF procedures. For RAD21-depletion analysis, cells were fixed for 10 mins at room temperature with $3.7 \%$ paraformaldehyde (Alfa Aesar) in PBS, washed 3 times with PBS and then permeabilized at room temperature for 10 mins with $0.25 \%$ Triton X-100 in PBS (Sigma Aldrich). For R-loop imaging, cells were fixed and permeabilised with ice-cold ultra-pure methanol (Sigma Aldrich) for 10 mins at $-20^{\circ} \mathrm{C}$. After 3 washes with PBS, cells were blocked for 45 mins at room temperature with $10 \%$ FCS-PBS. For RNASEH1 enzyme treatment, cells were incubated with blocking solution supplemented with 1x RNASEH1 reaction buffer alone $(50 \mathrm{mM}$ Tris- $\mathrm{HCl}, 75 \mathrm{mM} \mathrm{KCl}$, $3 \mathrm{mM} \mathrm{MgCl}_{2}, 10 \mathrm{mM}$ DTT) or 5 units of RNASEH1 enzyme (M0297, New England Biolabs) for 30 mins at $37^{\circ} \mathrm{C}$, PBS-washed twice, before blocking. Cells were washed twice with PBS before incubation with primary antibodies diluted in 5\% FCS-PBS at 4 ${ }^{\circ} \mathrm{C}$ overnight. Anti-SA1, anti-SA2 and anti-AQR were used at 1:3000 dilutions; antiCTCF was used at 1:2500 dilution; anti-s9.6 was used at 1:1000 dilution; anti-V5 was used at 1:1000. After 4 washes with PBS, cells were incubated with secondary antibodies (donkey anti-Goat AF555 or AF647 for SA1/2 used at 1:3000; donkey antiRabbit AF647 for CTCF used at 1:2500; donkey anti-Mouse AF555 for s9.6 used at 1:2000; donkey anti-Rabbit AF647 for AQR used at 1:3000; donkey anti-Rabbit AF488 for V5 used at 1:2000)) in 5\% FCS-PBS for 1 hour at room temperature, and washed 4 times with PBS before being mounted onto glass slides with ProLong ${ }^{\mathrm{TM}}$ Diamond Antifade Mountant with DAPI (Thermo Fisher Scientific) to stabilise overnight in dark before imaging. See Table 2 for details of where antibodies were purchased.

Imaging was performed on Zeiss LSM confocal microscopes using 63x/1.40 NA Oil Plan-Apochromat objective lens (Carl Zeiss, Inc.). Images were captured as zstacks and under consistent digital gain, laser intensity and resolution for each experiment. Numerical analysis was carried out using Imaris software (Oxford Instruments, version 9.5.1) and representative images are shown as maximum zprojected views generated using Fiji Image J. In brief, z-stack images were imported into Imaris, cells were identified using DAPI and only those located $1 \mu \mathrm{m}$ away from image boundary and sized between $120-800 \mu \mathrm{m}^{3}$ were selected. A seed-split function of 7.5um was used to separate closely situated cells. Fluorescence intensities of individual DAPI-selections in each channel were determined by Imaris and exported into Excel for further analysis. Distribution plots were generated from $>50$ cells of each replicate with 3 biological replicates per experiment. Student's $t$-test was performed between control and experimental conditions and statistical significance was determined by detecting the difference between means (unequal variance, two-tailed). 
895

896

897

898

899

900

901

902

903

904

905

906

907

908

909

910

911

912

913

914

915

916

917

918

919

920

921

922

923

924

925

926

927

928

929

930

931

932

933

934

935

936

Significance is denoted as $\mathrm{p}>0.05=$ not significant $(\mathrm{ns}), \mathrm{p} \leq 0.05={ }^{*}, \quad \mathrm{p} \leq 0.01=* *$, $\mathrm{p} \leq 0.001=^{* * *}$ and $\mathrm{p} \leq 0.0001{ }^{* * * *}$.

\section{Chromatin Fractionation and colmmunoprecipitation.}

Cells were washed twice with ice-cold PBS (Sigma Aldrich) and lysed in Buffer A (10 mM HEPES, 10mM KCl, $1.5 \mathrm{mM} \mathrm{MgCl2,} 0.34 \mathrm{M}$ Sucrose, 10\% Glycerol, 1mM DTT, $1 \mathrm{mM}$ PMSF/Pefabloc, protease inhibitor), supplemented with $0.1 \% \mathrm{~T}-\mathrm{X} 100$, for $10 \mathrm{~min}$ on ice. Lysed cells were collected by scraping. Nuclei and cytoplasmic material was separated by centrifugation for $4 \mathrm{~min}$ at $1300 \mathrm{~g}$ at $4 \mathrm{oC}$. The supernatant was collected as the cytoplasmic fraction and cleared of any insoluble material with further centrifugation for $15 \mathrm{~min}$ at $20,000 \mathrm{~g}$ at $40 \mathrm{C}$. The nuclear pellet was washed once with buffer A before lysis in buffer B (3mM EDTA, 0.2mM EGTA, 1mM DTT, 1mM PMSF/Pefabloc, protease inhibitor) with rotation for $30 \mathrm{~min}$ at $40 \mathrm{C}$. Insoluble nuclear material was spun down for $4 \mathrm{~min}$ at $1700 \mathrm{~g}$ at $4 \mathrm{oC}$ and the supernatant taken as nuclear soluble fraction. The insoluble material was wash once with buffer $B$ and then resuspended in high-salt chromatin solubilization buffer $(50 \mathrm{mM}$ Tris- $\mathrm{HCl} \mathrm{pH} 7.5,1.5$ $\mathrm{mM} \mathrm{MgCl}$, $500 \mathrm{mM} \mathrm{KCl,} \mathrm{1mM} \mathrm{EDTA,} \mathrm{20 \%} \mathrm{Glycerol,} \mathrm{0.1 \%} \mathrm{NP-40,} 1 \mathrm{mM}$ PMSF/Pefabloc, protease inhibitor). The lysate was vortexed for $2 \mathrm{~min}$ to aid solubilization. Nucleic acids were digested with $85 \mathrm{U}$ benzonase (Sigma-Aldrich) per $100 \times 106$ cells, with incubation for $10 \mathrm{~min}$ at $37 \mathrm{oC}$ and $20 \mathrm{~min}$ at $4 \mathrm{oC}$. Chromatin was further solubilized with ultra-sonication for $3 \times 10 \mathrm{sec}$ at an amplitude of 30 . The lysate was diluted to $200 \mathrm{mM} \mathrm{KCl}$ and insoluble material was removed by centrifugation at 15,000 RPM for $30 \mathrm{~min}$ at $40 \mathrm{C}$.

For colP, antibodies were bound to Dynabead Protein A/G beads (ThermoFisher Scientific) for $10 \mathrm{~min}$ at room temperature and $\sim \mathrm{hr}$ at $4 \mathrm{oC}$. For mock IgG IPs, beads were incubated with serum from the same host type as the antibody of interest. $1 \mathrm{mg}$ of chromatin extract was incubated with the antibody-bead conjugate per IP for approximately $16 \mathrm{hr}$ at $40 \mathrm{C}$. IPs were washed $\mathrm{x} 5$ with IP buffer $(200 \mathrm{mM}$ chromatin solubilization buffer) and eluted by boiling in either $2 x$ Laemmeli sample buffer (BioRad) or 4x NuPAGE LDS sample buffer (ThermoFisher Scientific). Proteins $\leq 250 \mathrm{kDa}$ were separated by SDS-PAGE electrophoresis using 4-20\% MiniPROTEAN® ${ }^{\circledR}$ TGX $^{\mathrm{TM}}$ Precast Protein Gels (BioRad) and transferred to Immobilon-P PVDF Membrane (Merck Millipore) for detection. Proteins $\geq 250 \mathrm{kDa}$ were separated by SDS-PAGE electrophoresis using Invitrogen NuPAGE 3-8\% Tris-Acetate precast protein gels. Transfer was extended to overnight with low voltage (20V) to aid in transfer of the high-molecular weight proteins. Membranes were incubated in primary antibody solution overnight at $4 \mathrm{OC}$ and images were detected using chemiluminescent fluorescence. Densitometry was carried out using ImageStudio Lite software with statistical significance calculated by unpaired t test, unless otherwise specified. Fold enrichment quantifications were performed by first normalising the raw densitometry value to its corresponding Histone $\mathrm{H} 3$ quantification and the comparing between the samples indicated. See Table 2 for details of antibodies. 


\section{S9.6 IP and Dot Blot.}

939 Cells were fractioned and processed for S9.6 IP as described above, with the following

940 modifications. To avoid digestion of RNA:DNA hybrids, samples were not treated

941 with benzonase during chromatin solubilization and sonication was carried out for 10

942 min (Diagenode Biorupter) as in ${ }^{34}$. Where indicated, chromatin samples were treated

943 with Ribonuclease $\mathrm{H}$ enzyme (NEB) overnight at $37^{\circ} \mathrm{C}$ to digest RNA:DNA hybrids in

944 the extract. To avoid detection of single-stranded RNA by the S9.6 antibody, all S9.6

945 IP samples were pre-treated with Purelink RNase A (Thermo Fisher Scientific) at

$9460.25 \mathrm{ug} / 1 \mathrm{mg}$ chromatin extract for $1 \mathrm{hr} 30 \mathrm{~min}$ at $4^{\circ} \mathrm{C}$. The reaction was stopped with

947 addition of $143 \mathrm{U}$ Invitrogen SUPERase-In RNase Inhibitor (Thermo Fisher

948 Scientific). RNA:DNA hybrid levels were assessed in chromatin samples by dot blot.

949 Specifically, the chromatin lysate was directly wicked

950 onto Amersham Protran nitrocellulose membrane (Merck) by pipetting small volumes

951 above the membrane. Membranes were blocked in $5 \%(w / v)$ non-fat dry milk in PBS-

$952 \quad 0.1 \%$ Tween and incubated with S9.6 antibody overnight as for standard western blot.

953 As above, detection was carried out using chemiluminescent fluorescence. RNase A-

954 mediated digestion of RNA:DNA hybrids was performed using a non-ssRNA-specific

955 enzyme (Thermo Scientific) at $1.5 \mathrm{ug} / 25 \mathrm{ug}$ chromatin extract at $37^{\circ} \mathrm{C}$.

956

957 ChIP-sequencing, library preparation and analysis.

958 ChIP lysates were prepared from RAD21 $1^{\mathrm{mAC}}$ cells treated with ethanol or IAA for

959 4hrs in two biological replicates. Formaldehyde (1\%) was added to the culture 960 medium for $10 \mathrm{~min}$ at room temperature. Fixation was blocked with $0.125 \mathrm{M}$ glycine and 961 cells were washed in cold PBS. Nuclear extracts were prepared by douncing (20 962 strokes, medium pestle) in swelling buffer (25 mM HEPES pH8, $1.5 \mathrm{mM} \mathrm{MgCl} 2,10 \mathrm{mM}$ $963 \mathrm{KCL}, 0.1 \%$ NP40, $1 \mathrm{mM}$ DTT and protease inhibitors) and centrifuged for $5 \mathrm{~min}$ at 964 2000rpm at 4C. Nuclear pellets were resuspended in Sucrose buffer I 965 (15mM Hepes pH 8, 340 mM Sucrose, 60mM KCL, 2mM EDTA, 0.5 mM EGTA, 0.5\% 966 BSA, $0.5 \mathrm{mM}$ DTT and protease inhibitors) and dounced again with 20 strokes. The 967 lysate was carefully laid on top of an equal volume of Sucrose buffer 968 II (15mM Hepes pH 8, 30\% Sucrose, 60mM KCL, 2mM EDTA, 0.5 mM EGTA, 0.5 mM 969 DTT and protease inhibitors) and centrifuged for $15 \mathrm{~min}$ at 4000rpm at 4C. Nuclei were 970 washed twice to remove cytoplasmic proteins, centrifuged and the pellet was 971 resuspended in Sonication/RIPA buffer (50mM Tris, $\mathrm{pH} 8.0,140 \mathrm{mM} \mathrm{NaCl}, 1 \mathrm{mM}$ 972 EDTA, 1\% Triton X-100, 0.1\% Na-deoxycholate, 0.1\% SDS and protease inhibitors) at 973 a concentration of $5 \times 10^{\wedge} 6$ nuclei in $130 \mu$ buffer. This was transferred to a sonication 974 tube (AFA Fiber Pre-Slit Snap-Cap 6x16mm) and sonicated in a Covaris S2 (settings; 9754 cycles of 60 seconds, $10 \%$ duty cycle, intensity: 5, 200 cycles per 976 burst). Soluable chromatin was in the range of $200-400 \mathrm{bp}$. Triton X100 was added 977 (final concentration 1\%) to the sonicated chromatin and moved to a low-retention 
tube (Eppendorf) before centrifugation at $14,000 \mathrm{rpm}$ for $15 \mathrm{~min}$ at $4 \mathrm{C}$ and pellets were discarded. $1 / 100^{\text {th }}$ of the chromatin lysate was retained as the Input sample.

For Immunoprecipitation, 200ug chromatin aliquots/IP were precleared with a slurry of Protein A/G (50:50) (Dynabeads) an incubated for $4 \mathrm{hr}$ at $4 \mathrm{C}$. Meanwhile, washed Protein $A / G$ beads (40ul per IP) were mixed with primary antibodies and incubated for $4 \mathrm{hrs}$ at $4 \mathrm{C}$. The following amounts of antibodies were used: antiCTCF, 5ug/ChIP; anti-SA1, 15ug/ChIP; anti-SA2, 10ug of the mixed antibody pack/ChIP; anti-Smc3, 5ug/ChIP and anti-lgG, 10ug/ChIP. See Table 2 for information about the antibodies. Washed, pre-bound Protein A/G beads+antibody were mixed with pre-cleared chromatin lysates and incubated overnight with rotation at $4 \mathrm{C}$. The next day, the supernatant was removed and the beads were washed 9 times with increasing salt concentrations. Protein-DNA crosslinks were reversed in ChIP elution buffer (1\% SDS, $5 \mathrm{mM}$ EDTA, $10 \mathrm{mM}$ Tris $\mathrm{HCl} \mathrm{pH} \mathrm{8)}+2.5$ ul of Proteinse $\mathrm{K}$ and incubated for 1 hour at $55^{\circ} \mathrm{C}$ and overnight at $65^{\circ} \mathrm{C}$. Samples were phenol-chloroform extracted, resuspended in TE buffer and assessed by qPCR as a quality control. Libraries were prepared from 5-10ng of purified DNA, depending on availability of material, using NEBNext Ultra II DNA Library Prep Kit for Illumina kit and using NEBNext Multiplex Oligos for Illumina (Index Primers Set 2) according to manufacturer's instructions using 6-8 cycles of PCR. ChIP-seq libraries from one biological set (all ChIP libraries for both ethanol and IAA) were multiplexed and sequenced on the Illumina HiSeq2500 platform, 80bp single-end reads. Each biological set was sequenced on a separate run.

Quality control of reads was preformed using FASTQC. Reads were aligned to the hg19 reference genome using Bowtie with 3 mismatches. Only replicate 1 of the SA1 librraye was used. PCR duplicates were detected and removed using SAMTOOLS. Bam files were imported into MISHA (v 3.5.6) and peaks were identified using a 0.995 percentile. Peaks that overlapped in both replicates were retained. Correlation plots of peaks across the genome from different ChIP libraries were compared with log-transformed percentiles plotted as a smoothed scatter plot. Comparison of peaks at regions of interest were carried out using deepTools (Version 3.1.0-2 REF). For input into deepTools, peak data was converted to bigwig format, with a bin size of 500 , using the UCSC bedGraphtoBigWig package. The signal matrix was calculated for a window 2,000 bp up- and down-stream of the region of interest, missing data was treated as zero, and all other parameters were as default. Heatmaps were generated within deepTools, with parameters as default.

\section{$\mathrm{Hi}-\mathrm{C}$ data and contact hotspots analysis.}

Generating hotspots - Previously published $\mathrm{Hi}-\mathrm{C}$ datasets derived from RAD2 $1^{\mathrm{mAC}}$ cells treated with ethanol or IAA ${ }^{8}$ were analyzed as previously described 51. Custom R scripts were written to identify Hi-C hotspots, i.e. regions of Hi-C maps with high contact frequency. To begin, for each chromosome, all contacts were extracted and subsetted for only high scoring $(>=60)$ contacts between a band of $10 \mathrm{e} 3$ 
$-70 \mathrm{e} 6$. Using KNN, for each high scoring contact, the 250 nearest neighbour contacts were identified and subset for only the high-scoring neighbours. This created a list of high scoring neighbours for each high scoring contact, where the first neighbour is the contact itself with a distance of 0 . This allowed the neighbour information to be converted into edge information, thereby allowing high score fend contacts to be grouped into cluster hotspots using the R package 'igraph'. Hotspots that contained less than the minimum number of high scoring fends $(<100)$ were removed. The output list of hotspots were represented as $2 \mathrm{D}$ intervals which contained high scoring contacts. In total, 5539 hotspots were identified in EtOH and 759 in IAA Hi-C data.

Creating aggregate plots - To calculate and visualise the contact enrichment at hotspots in the EtOH and IAA Hi-C, we used the R package 'shaman'. Firstly, we used the function 'shaman_generate_feature_grid' to calculate the enrichment profile at $\mathrm{EtOH}$ and IAA hotspots. Using the weighted centre for each hotspot, represented as a 2D interval we used the function to build grids for the $\mathrm{EtOH}$ and IAA hotspots in the $\mathrm{HiC}$ data at 3 specific bands, $100 \mathrm{k}-1 \mathrm{MB}, 1 \mathrm{MB}-5 \mathrm{MB}, 5 \mathrm{MB}-10 \mathrm{MB}$. A range of $250 \mathrm{~kb}$ was visualised around the weighted centre. The grid was built by taking all combinations interval1 and interval2 of the $\mathrm{EtOH}$ and IAA hotspot centres, with each combination termed a 'window'. Hotspots were not filtered for size or shape. A score threshold of 60 was used to focus on enriched pairs, those windows that did not contain at least one point with a score of 60 were discarded. Each window was then split into 1000nt bins and the windows were summed together to generate a grid containing the observed and expected contacts. We visualised the grid using 'shaman_plot_feature_grid' using 'enrichment' mode and a plot_resolution value of 6000 , due to the large range being visualised.

\section{STORM - Immunolabelling and imaging.}

Two clones of RAD2 $1^{\mathrm{mAC}}$-OsTIR cells were seeded at a density of 30,000 cells per well per 400ul) onto poly-L-lysine coated 8-well chamber slides (Lab-Tek ${ }^{\mathrm{TM}}$ 155411) overnight. Each clone was treated with either ethanol (EtOH) or Auxin (IAA) for $4 \mathrm{hr}$ and then fixed with PFA 4\% (Alfa Aesar) for $10 \mathrm{~min}$ at room temperature and rinsed with PBS three times for 5 min each. The cells were shipped to the Cosma Lab after fixation for STORM processing and imaging. Cells were permeabilized with $0.3 \%$ Triton X-100 in PBS and blocked in blocking buffer (10\% BSA - $0.01 \%$ Triton X-100 in PBS) for one hour at room temperature. Cells were incubated with primary antibodies (see Table 2) in blocking buffer at 1:50 dilution. Cells were washed three times for 5 min each with wash buffer (2\% BSA $-0.01 \%$ Triton X-100 in PBS) and incubated in secondary antibody. For STORM imaging, home-made (Bates et al., 2007) dye pair labeled secondary antibodies were added at a 1:50 dilution in blocking buffer and were incubated for $45 \mathrm{~min}$ at room temperature. Cells were washed three times for 5 min each with wash buffer. 

equipped with a CFI HP Apochromat TIRF 100x 1.49 oil objective and an iXon Ultra 897 camera (Andor) and using Highly Inclined and Laminated Optical sheet illumination (HILO). Dual color STORM imaging was performed with a double activator and single reporter strategy by combining AF405_AF647 anti-Goat secondary with

1066 Cy3_AF647 anti-Rabbit secondary antibodies. Sequential imaging acquisition was 1067 performed ( 1 frame of $405 \mathrm{~nm}$ activation followed by 3 frames of $647 \mathrm{~nm}$ reporter and 10681 frame of $560 \mathrm{~nm}$ activation followed by 3 frames of $647 \mathrm{~nm}$ reporter) with $10 \mathrm{~ms}$ exposure time for 120000 frames. $647 \mathrm{~nm}$ laser was used at constant 2 $\mathrm{kW} / \mathrm{cm}^{2}$ power density and $405 \mathrm{~nm}$ and $560 \mathrm{~nm}$ laser powers were gradually increased

1071 over the imaging. Imaging buffer composition for STORM imaging was $100 \mathrm{mM}$ 1072 Cysteamine MEA (Sigma-Aldrich, \#30070) - 5\% Glucose (Sigma-Aldrich, \#G8270) $10731 \%$ Glox Solution $(0.5 \mathrm{mg} / \mathrm{ml}$ glucose oxidase, $40 \mathrm{mg} / \mathrm{ml}$ catalase (Sigma-Aldrich, \#G2133 and \#C100)) in PBS.

\section{STORM imaging analysis and quantifications.}

1077 STORM images were analyzed and rendered in Insight3 (kind gift of Bo Huang, UCSF) as previously described (Bates et al., 2007; Rust et al., 2006). Localizations were identified based on a threshold and fit to a simple Gaussian to determine the $\mathrm{x}$ and $\mathrm{y}$ positions. Cluster analysis of CTCF, SA1 and SA2 STORM signal was performed as previously described (Ricci et al., 2015) to obtain cluster size and positions and to measure Nearest Neighbour distributions (NND) between clusters of the same protein in individual nuclei. NND between clusters' centroids of two different proteins (i.e. CTCF-SA1 and CTCF-SA2) was calculated by knnsearch.m Matlab function and the NND histogram of experimental data was obtained by considering all the NNDs of individual nuclei (histogram bin, from 0 to $500 \mathrm{~nm}, 5 \mathrm{~nm}$ steps). Simulated NNDs recapitulating random spatial distribution of cluster centroids were first obtained for each nucleus separately and then merged to calculate the simulated NND histogram (histogram bin, from 0 to $500 \mathrm{~nm}, 5 \mathrm{~nm}$ steps). The difference plot reports the difference between experimental NND and simulated NND. Quantification and analysis of STORM images was performed in Matlab and statistical analysis was performed in Graphpad Prism (v7.0e). The type of statistical test is specified in each case. Statistical significance is represented as indicated above.

1094 Insight3 Software used for STORM image processing has been generated (Huang et al., 2008) and kindly provided by Dr Bo Huang (UCSF). Graphpad Prism software used for statistical analysis can be found at: https://www.graphpad.com/scientificsoftware/prism/ MatLab software used for imaging data analysis can be found at: https://www.mathworks.com/products/matlab.html

1101 SA1 immunoprecipitation samples were analysed by liquid chromatography-tandem 1102 mass spectrometry (LC-MS/MS). Three biological replicate experiments were carried 
1103 out for MS and each included four samples, untreated (UT), treated with IAA for

1104 4hrs, siCon, or siSA1, generated as described above. A fourth technical replicate was 1105 also included for the UTR samples. Cells were fractionated to purify chromatin-bound 1106 proteins as above and immunoprecipitated with IgG- or SA1-bead 1107 conjugates. To maximise IP material for the MS, the antibody amount was increased 1108 to $15 \mathrm{ug}$ and the chromatin amount was increased to $2 \mathrm{mg}$.

1109 The IP eluates were loaded into a pre-cast SDS-PAGE gel (4-20\% Mini1110 PROTEAN® ${ }^{\circledR}$ TGX $^{\mathrm{TM}}$ Precast Protein Gel, 10-well, $50 \mu \mathrm{L}$ ) and proteins were run 1111 approximately $1 \mathrm{~cm}$ to prevent protein separation. Protein bands were excised and 1112 diced, and proteins were reduced with $5 \mathrm{mM}$ TCEP in $50 \mathrm{mM}$ triethylammonium 1113 bicarbonate (TEAB) at $37^{\circ} \mathrm{C}$ for $20 \mathrm{~min}$, alkylated with $10 \mathrm{mM}$ 2-chloroacetamide in 50 $1114 \mathrm{mM}$ TEAB at ambient temperature for $20 \mathrm{~min}$ in the dark. Proteins were then digested 1115 with $150 \mathrm{ng}$ trypsin, at $37^{\circ} \mathrm{C}$ for $3 \mathrm{~h}$ followed by a second trypsin addition for $4 \mathrm{~h}$, then 1116 overnight at room temperature. After digestion, peptides were extracted with 1117 acetonitrile and $50 \mathrm{mM}$ TEAB washes. Samples were evaporated to dryness at $30^{\circ} \mathrm{C}$ 1118 and resolubilised in $0.1 \%$ formic acid.

1119 nLC-MS/MS was performed on a $Q$ Exactive Orbitrap Plus interfaced to a 1120 NANOSPRAY FLEX ion source and coupled to an Easy-nLC 1200 (Thermo Scientific). $112125 \%$ (first, second and fourth biological replicate) or 50\% (third biological replicate) of 1122 each sample was loaded as 5 or $10 \mu \mathrm{L}$ injections. Peptides were separated on a $27 \mathrm{~cm}$

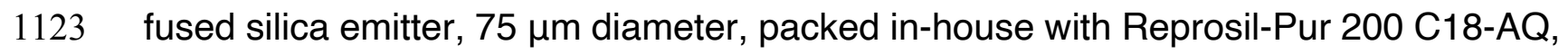
$11242.4 \mu \mathrm{m}$ resin (Dr. Maisch) using a linear gradient from 5\% to 30\% acetonitrile/ $0.1 \%$ 1125 formic acid over $60 \mathrm{~min}$, at a flow rate of $250 \mathrm{~nL} / \mathrm{min}$. Peptides were ionised by 1126 electrospray ionisation using $1.8 \mathrm{kV}$ applied immediately prior to the analytical column 1127 via a microtee built into the nanospray source with the ion transfer tube heated to $1128320^{\circ} \mathrm{C}$ and the S-lens set to $60 \%$. Precursor ions were measured in a data-dependent 1129 mode in the orbitrap analyser at a resolution of 70,000 and a target value of 3e6 ions. 1130 The ten most intense ions from each MS1 scan were isolated, fragmented in the HCD 1131 cell, and measured in the orbitrap at a resolution of 17,500.

1132

1133 Mass spectrometry analysis

1134 Raw data was analysed with MaxQuant ${ }^{52}$ version 1.5.5.1 where they were searched 1135 against the human UniProtKB database using default settings 1136 (http://www.uniprot.org/). Carbamidomethylation of cysteines was set as fixed 1137 modification, and oxidation of methionines and acetylation at protein $\mathrm{N}$-termini were 1138 set as variable modifications. Enzyme specificity was set to trypsin with maximally 2 1139 missed cleavages allowed. To ensure high confidence identifications, PSMs, peptides, 1140 and proteins were filtered at a less than $1 \%$ false discovery rate (FDR). Label-free 1141 quantification in MaxQuant was used with LFQ minimum ratio count set to 2 with 1142 'FastLFQ' (LFQ minimum number of neighbours = 3, and LFQ average number of 1143 neighbours $=6$ ) and 'Skip normalisation' selected. In Advanced identifications, 1144 'Second peptides' was selected and the 'match between runs' feature was not 
selected. Statistical protein quantification analysis was done in MSstats ${ }^{53}$ (version 3.14.0) run through RStudio. Contaminants and reverse sequences were removed and data was log2 transformed. To find differential abundant proteins across conditions, paired significance analysis consisting of fitting a statistical model and performing model-based comparison of conditions. The group comparison function was employed to test for differential abundance between conditions. Unadjusted pvalues were used to rank the testing results and to define regulated proteins between 1152 groups.

Proteins with peptides discovered in the IgG samples were disregarded from downstream analyses. Significantly depleted/enriched proteins were considered with an absolute log2foldchange $>0.58$ (1.5-fold change) and a p-value $<0.1$. SA1 interactome analysis was performed in STRING. The network was generated as a full STRING network with a minimum interaction score of 0.7 required. Over-enrichment of GO biological process and molecular function terms was calculated with the human genome as background. Network analysis of the SA1 interactome in IAA-treated samples was generated from the significantly depleted/enriched proteins, with a minimum interaction score of 0.4 required. Two conditions for functional enrichments were considered; i) enrichment was calculated with the human genome as background to determine the full SA1 interactome in the absence of cohesin, compared to the genome, and ii) enrichment was calculated with the untreated SA1 interactome as background, to determine the statistical effect of cohesin loss of the SA1 interactome itself. The network developed in i) was manually rearranged in Cytoscape for visual clarity, enriched categories were visualized using the STRING pie chart function and half of the proteins within each category were subset from the network based on pvalue change between UTR and IAA samples.

Over-enrichment of the $\$ 9.6$ interactome was calculated separately using the hypergeometric distribution for comparison with ${ }^{34,35}$. Significance was calculated using the dhyper function in $\mathrm{R}$ and multiple testing was corrected for using the p.adjust Benjamini \& Hochberg method. To compare with a minimal background protein list, http://www.humanproteomemap.org was analysed on the Expression Atlas database to determine a list of proteins expressed in one or more of three tissue types corresponding to the cell types used across the different studies.

\section{SLiMSearch analysis}

1180 The SLiMSearch tool http://slim.icr.ac.uk/slimsearch/, with default parameters was used to search the human proteome for additional proteins that contained the FGFlike motif determined in ${ }^{16}$ to predict binding to SA proteins. The motif was input as [PFCAVIYL][FY][GDEN]F.\{0,1\}[DANE].\{0,1\}[DE]. Along with CTCF, four proteins

1184 found to contain the FGF-like motif, CHD6, MCM3, HNRNPUL2 and ESYT2 were validated for interaction with SA. 


\section{CLIP and iCLIP}

1189 Crosslinking immunoprecipitation (CLIP) was performed as previously described ${ }^{54}$.

1190 Briefly, mESC or HCT116 cells were irradiated with $0.2 \mathrm{~J} / \mathrm{cm} 2$ of $254 \mathrm{~nm}$ UV light in a 1191 Stratalinker 2400 (Stratagene). Cells were lysed in $1 \mathrm{ml}$ of lysis buffer with Complete 1192 protease inhibitor (Roche). Lysates were passed through a $27 \mathrm{G}$ needle, 1.6 U DNase 1193 Turbo (Thermofisher) per $10^{6}$ cells and 0.8 (low) or $8 \cup$ (high) U RNase I (Ambion) per $119410^{6}$ cells added, and incubated in a thermomixer at $37^{\circ} \mathrm{C}$ and $1100 \mathrm{rpm}$ for 3 minutes. 1195 Lysates were then cleared by centrifugation and using Proteus clarification spin 1196 column, according to the manufacturer's instructions. Endogenous SA1 and SA2 were 1197 immunoprecipitated with 10 g SA1 and SA2 antibodies or non-specific IgG control 1198 (Sigma) conjugated to protein $\mathrm{G}$ dynabeads (Dynal) for $4 \mathrm{hrs}$ at $4^{\circ} \mathrm{C}$. Tagged SA2 1199 proteins were immunoprecipitated from HCT116 cells 40 hours after transfection with 120030 I GFP-Trap beads. IPs were washed three times with high salt buffer (containing $12011 \mathrm{M} \mathrm{NaCl}$ and $1 \mathrm{M}$ urea) and once with PNK buffer and RNA labelled with $8 \mu \mathrm{l}$ 1202 radioactive ${ }^{32} \mathrm{P}$-gamma-ATP (Hartmann Analytic) for 5 mins at $37^{\circ} \mathrm{C}$. For $\mathrm{RNaseH} 1$ 1203 treatment, YFP-SA2 samples were split and either treated with PNK buffer alone or 1204 PNK buffer containing $50 \mathrm{U}$ RNaseH1 for 15 mins at $37^{\circ} \mathrm{C}$. RNPs were eluted in LDS 1205 loading buffer (Invitrogen) and resolved on a 4-12\% gradient NuPAGE Bis-Tris gel 1206 (Invitrogen) and transferred onto $0.2 \mu$ m diameter pore nitrocellulose membrane. After 1207 blocking with PBST+milk, membranes were washed and exposed overnight to 1208 phosphorimager screen (Fuji) and $\mathrm{RNA}^{32} \mathrm{P}$ visualized using a Typhoon 1209 phosphorimager (GE) and ImageQuant TL (GE). Membranes were then 1210 immunoblotted for SA1, SA2, and RAD21 and visualized using an ImageQuantLAS 12114000 imager (GE). See Table 2 for details on antibodies.

1212

1213 GFP-TRAP + Cloning of STAG2 s/I and YFP constructs.

1214 SA2 cDNAs were cloned directly from HCT116 cells by PCR using KAPA 1215 HiFi HotStart PCR kit (Roche) (Fwd: ATGATAGCAGCTCCAGAAAACCAACTG; Rev: 1216 TTAAAACATTGACACTCCAAGAACTGATTCATCC). Two major isoforms were 1217 detected, $\mathrm{SA} 2^{\Delta \mathrm{ex} 32}$ where exon32 has been spliced out and $\mathrm{SA} 2^{+\mathrm{ex} 32}$ where exon 121832 has been spliced in. Both SA2 cDNAs were cloned into pENTR/D vector 1219 (Invitrogen) and then into an N-terminal YFP-tagged Gateway cloning vector (a kind 1220 gift from Endre Kiss-Toth, University of Sheffield). Sequences were confirmed by restriction enzyme digestion and Sanger sequencing. Recombinant YFP-SA2 ${ }^{\triangle \mathrm{ex} 32}$ or

1222 YFP-SA2 ${ }^{+\mathrm{ex} 32}$ were transfected into adherent HCT116 cells for 40 hours before being 1223 harvested. Cells were lysed, fractionated and sonicated following the same protocol 1224 for chromatin fractionation with the variation of chromatin solubilisation in $\mathrm{NaCl}$ IP 1225 buffer (50mM Tris-HCL pH 7.5, 150mM NaCl, 1mM EDTA, 0.1\% NP-40, 20\% Glycerol, $12261 \mathrm{mM}$ DTT). 1mg chromatin lysate was pre-cleared with a 50:50 mixture of protein A/G 1227 magnetic beads and GFP-Trap (Chromotek, gtd-20) was pre-blocked with $1 \mathrm{mg} / \mathrm{mL}$ 1228 ultra-pure BSA (AM2616, Invitrogen) for $2 \mathrm{~h}$ at $4^{\circ} \mathrm{C}$. After blocking, GFP-Trap was 
1229 washed twice with $\mathrm{NaCl}$ IP buffer and added to pre-cleared lysates to

1230 immunoprecipitate proteins for $1 \mathrm{~h}$ at $4^{\circ} \mathrm{C}$. Samples were washed in NaCI IP buffer and

1231 eluted in 2x Laemmli buffer (Bio-Rad). Proteins were separated by SDS-PAGE on a

1232 4-20\% gradient mini-PROTEAN® Precast Gel (Bio-Rad) and transferred onto PVDF

1233 membrane for visualization.

1234

1235 VAST-TOOLS

1236 VAST-TOOLS was used to generate Percent Spliced In (PSI) scores, a statistic which

1237 represents how often a particular exon is spliced into a transcript using the ratio

1238 between reads which include and exclude said exon. Paired-end RNA-seq datasets

1239 were submitted to VAST-TOOLS (v2.1.3) using the Mmu genome (Tapial J et al, Gen

1240 Res 2017). Briefly, reads are split into 50nt words with a 25nt sliding window. The 50nt

1241 words are aligned to a reference genome using Bowtie to obtain unmapped reads.

1242 These unmapped reads are then aligned to a set of predefined exon-exon junction

1243 (EJJ) libraries allowing for the quantification of alternative exon events. The output

1244 was further interrogated using a script which searches all hypothetical EEJ

1245 combinations between potential donors and acceptors within Stag1. PSI scores could

1246 be obtained providing there was at least a single read within the RNAseq data that

1247 supported the event, although we only considered events supported by a minimum

1248 of 50 reads. Calculated PSI values for each alternatively spliced exon (and shown in

1249 Fig 3d), as well as the average PSI reported in the text are shown below. See

1250 Table 3 for names of published datasets used in this analysis.

1251

\begin{tabular}{|l|l|l|l|l|}
\hline Dataset & $\begin{array}{l}\text { SA2 } \\
\text { e32 PSI }\end{array}$ & Reads across junction & $\begin{array}{l}\text { SA1 } \\
\text { e31 PSI }\end{array}$ & Reads across junction \\
\hline ENCODE HCT & 21.92 & 202.98 & 98.99 & 588 \\
\hline Zuo HCT & 19.18 & 94.75 & 97.91 & 278.06 \\
\hline ENCODE HeLa & 19.95 & 90.57 & 96.36 & 156.1 \\
\hline AVG & $\mathbf{2 0 . 3 5}$ & & $\mathbf{9 7 . 7 5}$ & \\
\hline
\end{tabular}


1254 Table 1. siRNAs used in this study.

\begin{tabular}{|l|l|l|l|l|}
\hline siRNA name & Company & Target & Catalogue no. & custom siRNA sequence \\
\hline si scramble control & Dharmacon & Smartpool & D-001810-10-05 & \\
\hline siSA1 & Dharmacon & Smartpool & L-010638-01-0010 & \\
\hline siSA2 & Dharmacon & Smartpool & L-021351-00-0010 & \\
\hline siNIPBL & Dharmacon & Smartpool & L-012980-00-0010 & \\
\hline siAQR & Dharmacon & Smartpool & L-022214-01-0005 & \\
\hline siControl (scramble) & Dharmacon & Smartpool & D-001810-10-20 & \\
\hline esi control & Sigma & Luciferase & EHUFLUC & \\
\hline esi SA1 & Sigma & exon 31 & custom esiRNA & $\begin{array}{l}\text { TCCTCAGATGCAGATCTCTTGGTTAGGCC } \\
\text { AGCCGAAGTTAGAAGACTAAATCGGAAG } \\
\text { GACAGAACAGGAATGAACTACATGAAAGTG } \\
\text { AGAACTGGAGGAGGCATGCTGT }\end{array}$ \\
\hline esi SA2 & Sigma & exon 32 & custom esiRNA & $\begin{array}{l}\text { CACGCAGGTAACATGGATGTTAGCTCAAGG } \\
\text { ACAACAGGAGGAGCAAGGCAACAGCAGG } \\
\text { AGAGAGCAGCAATGAGCTATGTAAACTG } \\
\text { CGAACTAATCTTCAGCATGCCAT }\end{array}$ \\
\hline
\end{tabular}

Table 2. Antibodies used in this study.

\begin{tabular}{|c|c|c|c|c|}
\hline Protein & Company & Catalogue No. & Species & $\begin{array}{l}\text { Figure } \\
\text { References }\end{array}$ \\
\hline SA1 & Abcam & ab4455 & mouse & $\begin{array}{l}1 a, c, d, e, 2 a, b, d \\
3 a, b, e, 4 b, c, e, 5 d\end{array}$ \\
\hline SA1 & Abcam & ab4457 & mouse & $1 \mathrm{i}$ \\
\hline SA2 & Bethyl & A300-159 & goat & $\begin{array}{l}1 \mathrm{~b}, \mathrm{c}, \mathrm{d}, \mathrm{e}, 2 \mathrm{a}, 3 \mathrm{a}, \mathrm{b} \\
\mathrm{f}, 4 \mathrm{c}, \mathrm{e}, 5 \mathrm{~d}\end{array}$ \\
\hline SA2 & Bethyl, AbVantage Pack & A310-941A & goat & $1 \mathrm{i}$ \\
\hline CTCF & Diagenode & C15410210 & rabbit & $1 \mathrm{c}, \mathrm{d}, \mathrm{i}, 2 \mathrm{a}$ \\
\hline CTCF & Cell signalling & 2899s & rabbit & $1 \mathrm{a}, \mathrm{e}$ \\
\hline Rad21 & Abcam & ab992 & rabbit & $\begin{array}{l}1 \mathrm{c}, \mathrm{d}, \mathrm{i}, 2 \mathrm{a}, \mathrm{d}, 5 \mathrm{~b}, \\
\mathrm{~d}, \mathrm{f}\end{array}$ \\
\hline GFP-TRAP & ChromoTek & gtd-20 & & $1 \mathrm{i}, 3 \mathrm{~g}$ \\
\hline GFP & Invitrogen & A11122 & rabbit & $1 \mathrm{a}, \mathrm{e}$ \\
\hline mAID & MBL & M214-3 & mouse & s1a \\
\hline OsTIR & MBL & PD048 & rabbit & s1a \\
\hline Smc3 & Abcam & ab9263 & rabbit & $1 \mathrm{i}$ \\
\hline CHD6 & Bethyl & A301-221A & rabbit & $2 a$ \\
\hline Mcm3 & Bethyl & A300-124A & goat & $2 a, 4 b$ \\
\hline HNRNPUL2 & Abcam & ab195338 & rabbit & $2 a$ \\
\hline YTHDC1 & Abcam & ab122340 & rabbit & $2 d$ \\
\hline FTSJ3 & Bethyl & А304-199A-M & rabbit & $2 d$ \\
\hline FANCI & Bethyl & A301-254A-M & rabbit & $2 d$ \\
\hline TAF15 & Abcam & ab134916 & rabbit & $2 d$ \\
\hline DHX9 & Abcam & Ab26271 & rabbit & $2 d, 4 b$ \\
\hline SSRP1 & Abcam & ab26212 & mouse & $2 \mathrm{~d}$ \\
\hline INO80 & Proteintech & 18810-1-AP & rabbit & $2 d$ \\
\hline ESYT2 & Sigma-Aldrich & HPA002132 & rabbit & $2 d$ \\
\hline S9.6 & Kerafast & ENH001 & mouse & $4 b, c, e, 5 f$ \\
\hline RNase H2 & Novus & NBP1-76981 & rabbit & $4 \mathrm{~b}$ \\
\hline AQR & Bethyl & A302-547A & rabbit & $4 b, e, 5 f$ \\
\hline Pol2 & Covance & MMS-1289 & mouse & $4 b$ \\
\hline Mau2 & Abcam & $a b 183033$ & rabbit & $5 b, d, f$ \\
\hline NIPBL & Abbiotec & 250133 & rat & $5 b, d, f, S 5$ \\
\hline $\mathrm{H} 3$ & Abcam & ab1791 & rabbit & $3 e, f, 5 b, d, f$ \\
\hline
\end{tabular}




\begin{tabular}{|l|l|l|l|l|}
$\begin{array}{l}\text { Name (Secondar } \\
\text { y Abs) }\end{array}$ & Fluorophore & Company & $\begin{array}{l}\text { Catalogue } \\
\text { No. }\end{array}$ & $\begin{array}{l}\text { Figure } \\
\text { References }\end{array}$ \\
\hline $\begin{array}{l}\text { Donkey anti- } \\
\text { Rabbit }\end{array}$ & Cy3_AF647 & $\begin{array}{l}\text { Home made from } \\
\text { Jackson Immunores } \\
\text { earch IgG }\end{array}$ & $\begin{array}{l}\text { Home } \\
\text { made from } \\
711-005- \\
152\end{array}$ & $1 \mathrm{e}, \mathrm{S} 1$ \\
& & $\begin{array}{l}\text { Home made from } \\
\text { lackson Immunores } \\
\text { earch IgG }\end{array}$ & $\begin{array}{l}\text { Home } \\
\text { made from } \\
705-005- \\
147\end{array}$ & \\
\hline Donkey anti-Goat & AF405_AF647 & Ie, S1 & \\
& & Invitrogen & A31570 & $1 \mathrm{a}, \mathrm{d}, \mathrm{e}$ \\
\hline Donkey anti-mouse & AF647 & Invitrogen & A21206 & $1 \mathrm{a}, \mathrm{d}, \mathrm{e}$ \\
\hline Donkey anti-rabbit & AF488 & Invitrogen & A31573 & $1 \mathrm{a}, \mathrm{d}, \mathrm{e}$ \\
\hline Donkey anti-rabbit & AF647 & Invitrogen & A21432 & $1 \mathrm{a}, \mathrm{d}, \mathrm{e}$ \\
\hline Donkey anti-goat & AF555 & Invitrogen & A21447 & $1 \mathrm{a}, \mathrm{d}, \mathrm{e}$ \\
\hline Donkey anti-goat & AF647 & &
\end{tabular}

1259 Table 3. Published datasets used in this study.

\begin{tabular}{|l|l|l|l|}
\hline Accession no. & Analysis description & Publication DOI or Ref & $\begin{array}{l}\text { Figure } \\
\text { Reference }\end{array}$ \\
\hline GSE104334 & $\begin{array}{l}\text { Long-range contact analysis of } \\
\text { Hi-C datasets }\end{array}$ & $10.1016 /$ j.cell.2017.09.026 & $1 \mathrm{i}$ \\
\hline GSE89729 & $\begin{array}{l}\text { Percent Spliced In (PSI) analysis } \\
\text { ff RNA-seq datasets }\end{array}$ & $10.1172 /$ jci.insight.91419 & 4d, "HCT Zuo" \\
\hline GSM958749 & $\begin{array}{l}\text { Percent Spliced In (PSI) analysis } \\
\text { of RNA-seq datasets }\end{array}$ & ENCODE HCT116 RNAseq & $\begin{array}{l}\text { 4d, "HCT } \\
\text { ENCODE" }\end{array}$ \\
\hline GSM958735 & $\begin{array}{l}\text { Percent Spliced In (PSI) analysis } \\
\text { of RNA-seq datasets }\end{array}$ & ENCODE HeLa RNAseq & 4d, "HeLa" \\
\hline
\end{tabular}


bioRxiv preprint doi: https://doi.org/10.1101/2021.02.20.432055; this version posted February 21, 2021. The copyright holder for this Figure 1. (which was not certified by peer review) is the author/funder, who has granted bioRxiv a license to display the preprint in
perpetuity. It is made available under aCC-BY 4.0 International license.
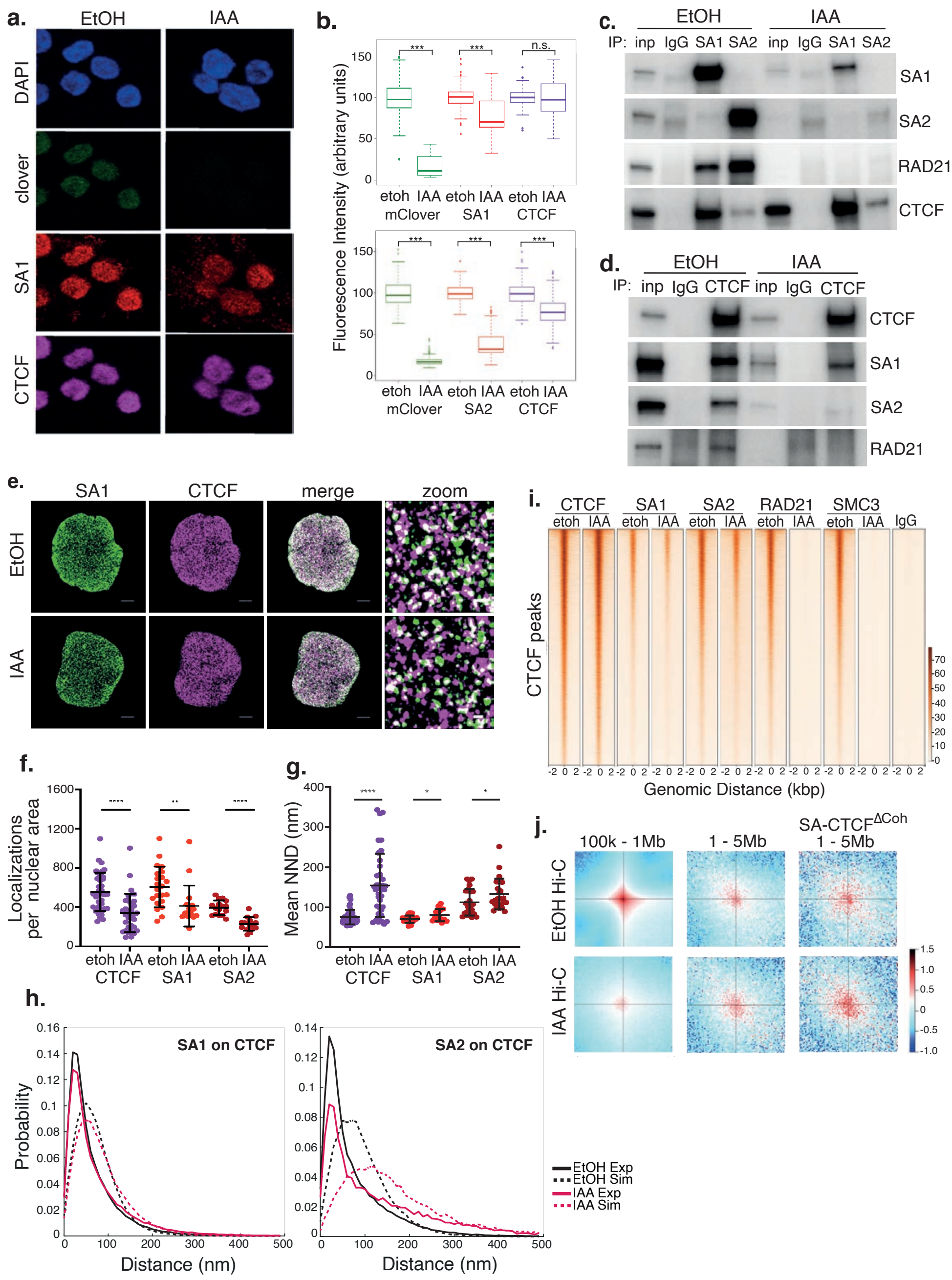

Genomic Distance (kbp)

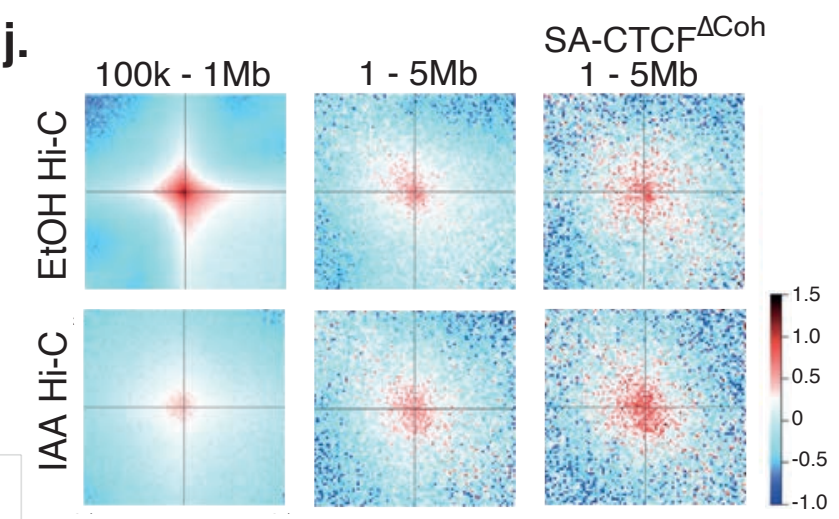


bioRxiv preprint doi: https://doi.org/10.1101/2021.02 20.432055 - this version posted February 21, 2021. The copyright holder for this Freprint (which was not certified by peer review) is the author/funder, who has granted bioRxiv a license to display the preprint in Figure 2.

a.
$\mathrm{EtOH}$
IAA
IP: inp lgG SA1 SA2 inp lgG SA1SA2

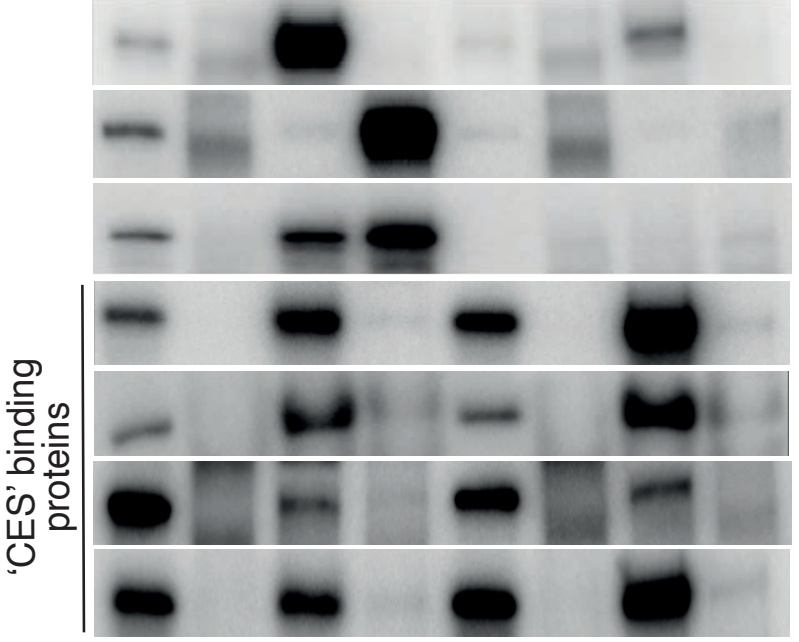

SA1

SA2

$\operatorname{Rad} 21$

CTCF

CHD6

$\mathrm{MCM} 3$

hnRNPUL2 b.

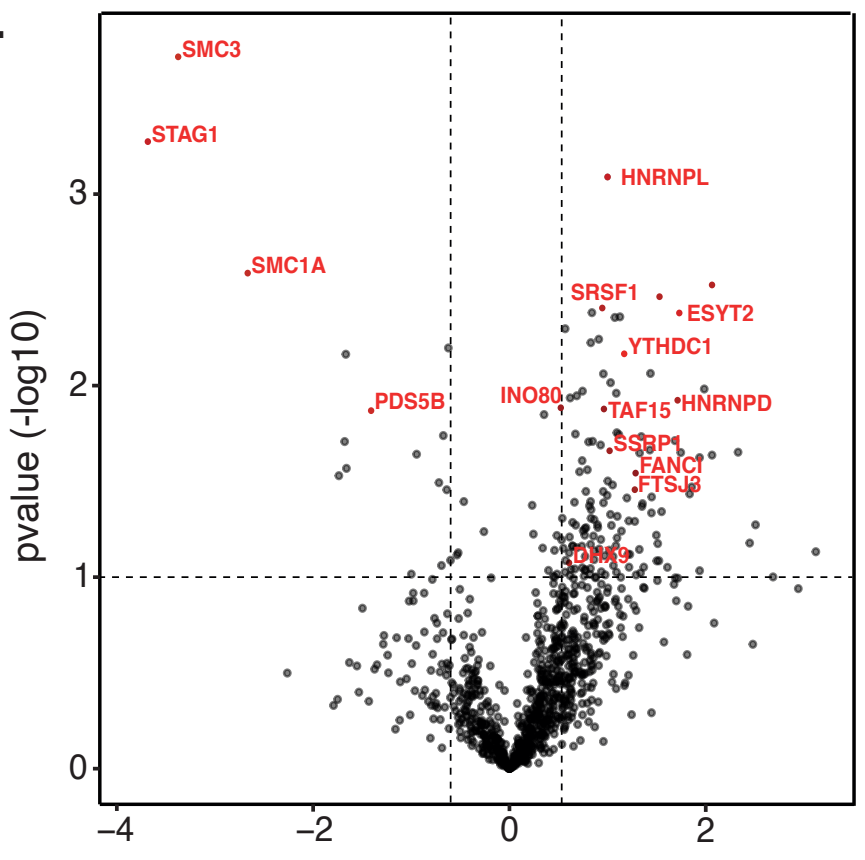

Fold Difference IAA-EtOH (log2)

C.

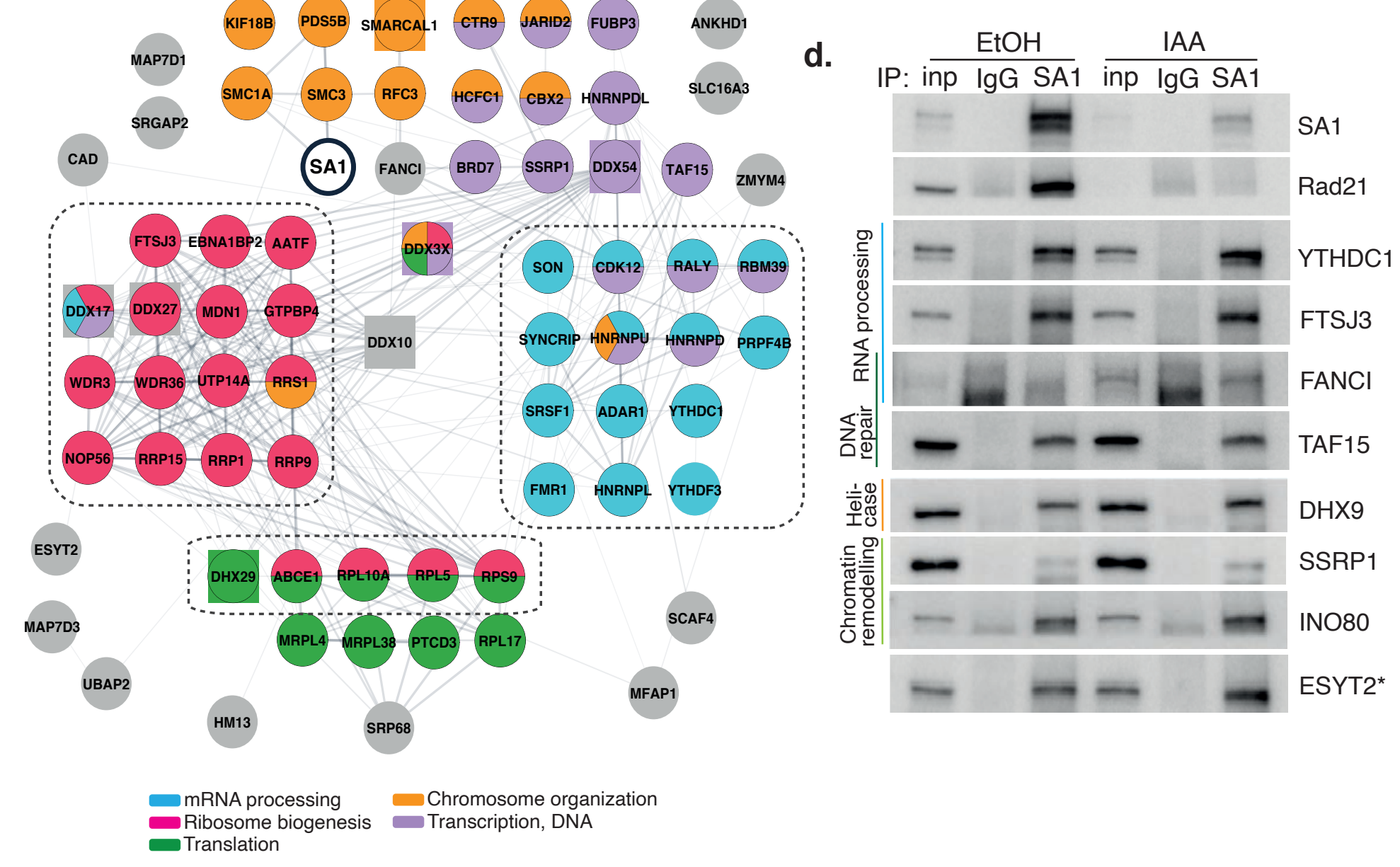


bioRxiv preprint doi: https://doi.org/10.1101/2021.02.20.432055; this version posted February 21, 2021. The copyright holder for this preprint (which was not certified by peer review) is the author/funder, who has granted bioRxiv a license to display the preprint in

Figure 3.

a.

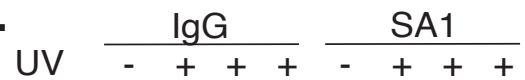

PNK +-+++-++

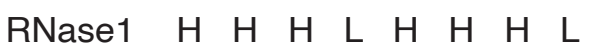
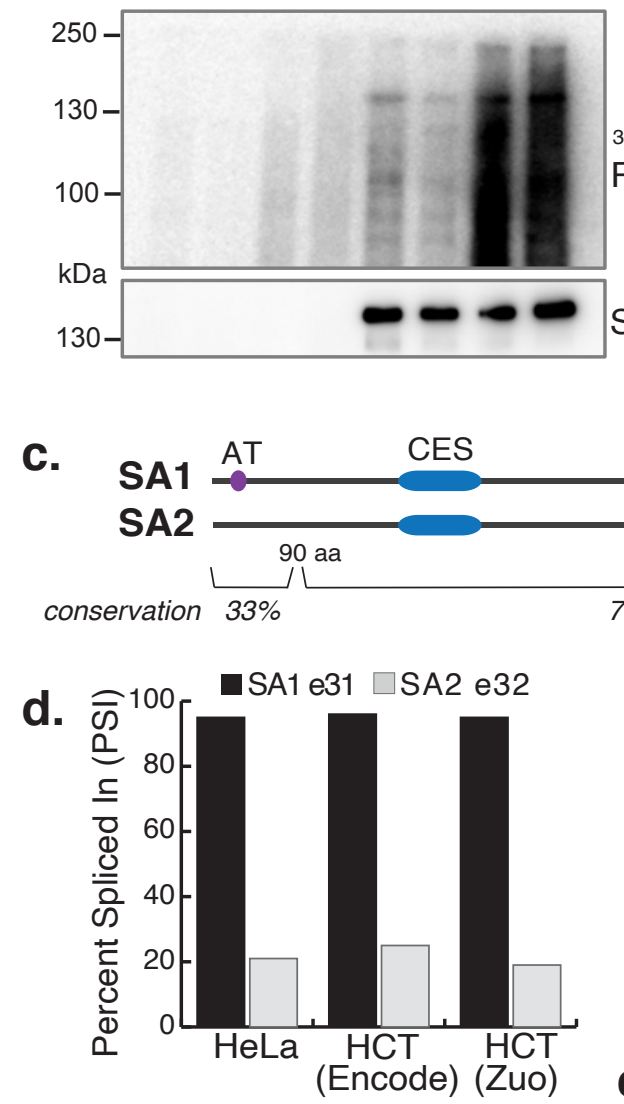
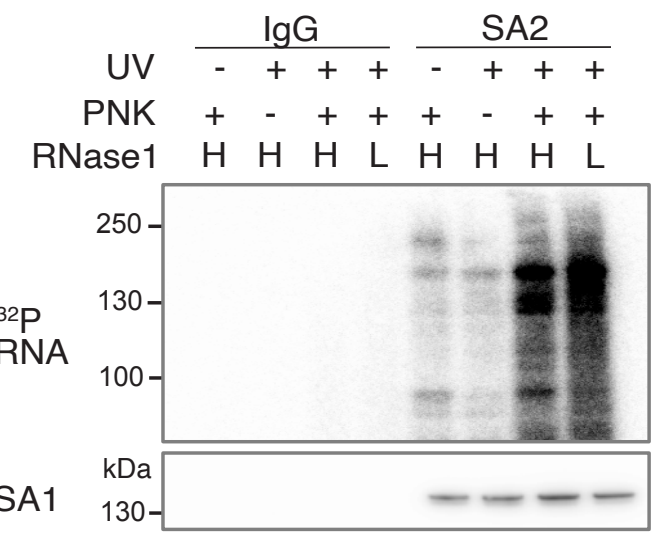

SA2 b.

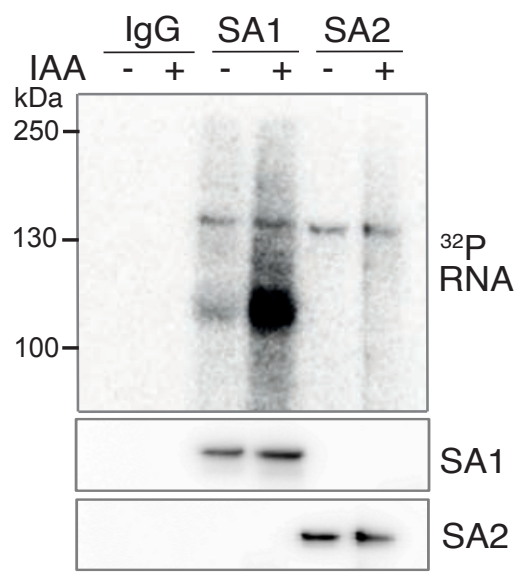

g.

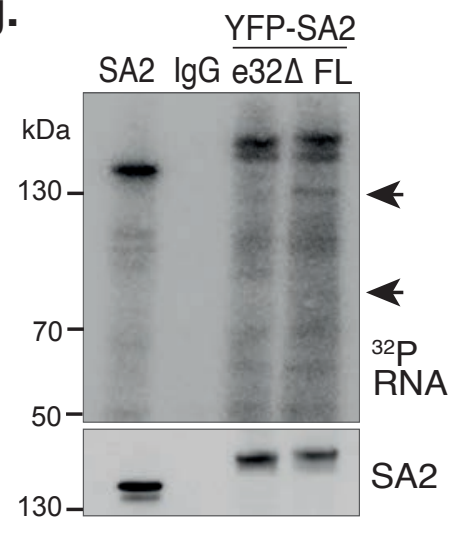

e. con siSA1 esi esiSA1

f. con siSA2 esi esiSA2

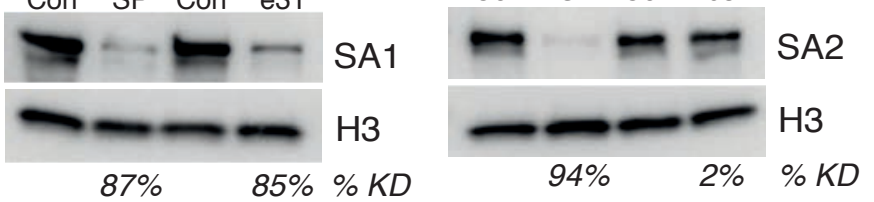


a.

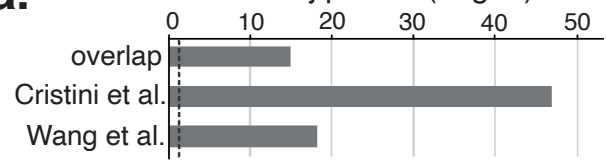

b.

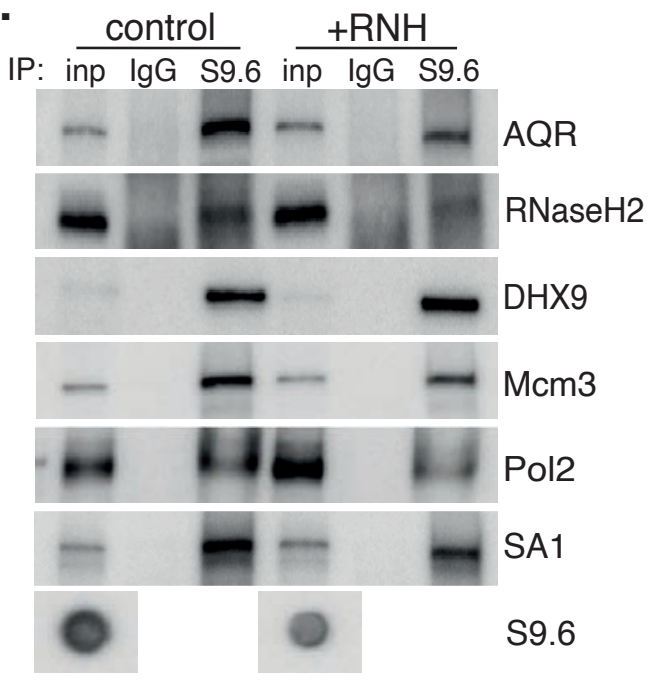

C.

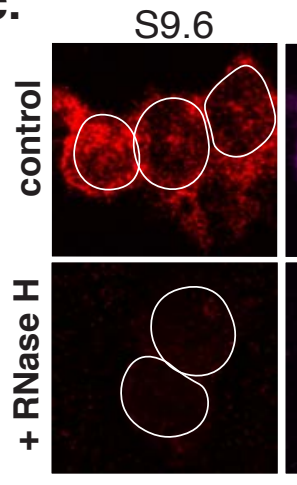

d.

\section{d.}

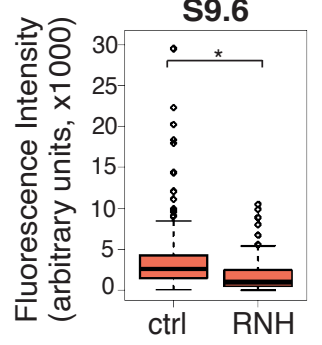

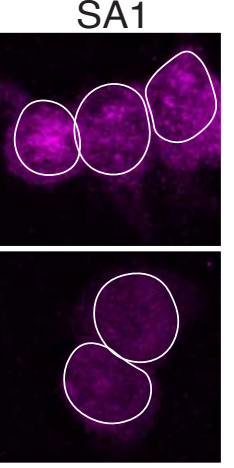

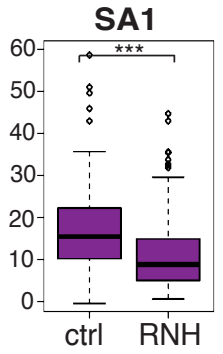

S9.6

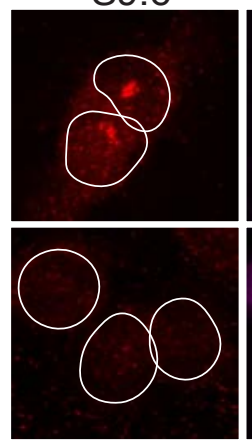

S9.6

SA2

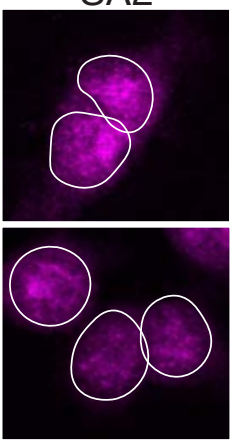

SA2
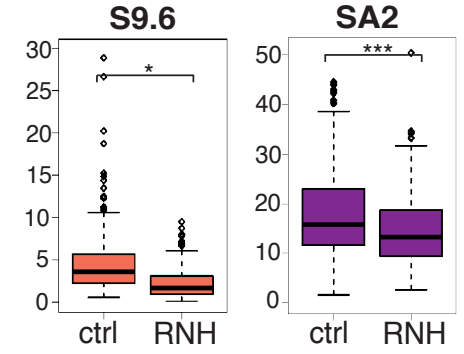

e.

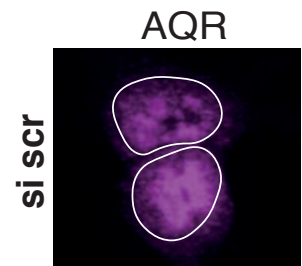

S9.6

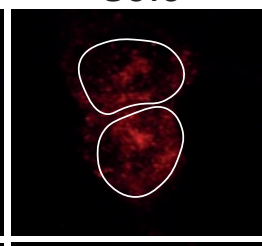

SA1

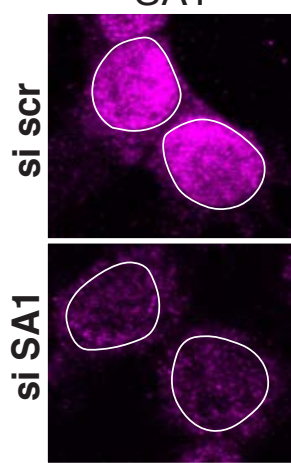

S9.6
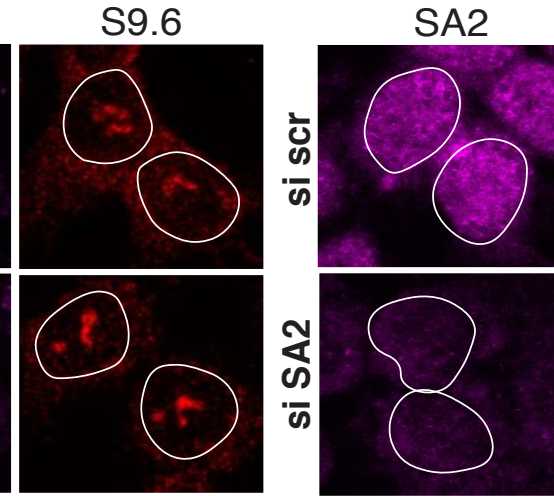

S9.6

f.
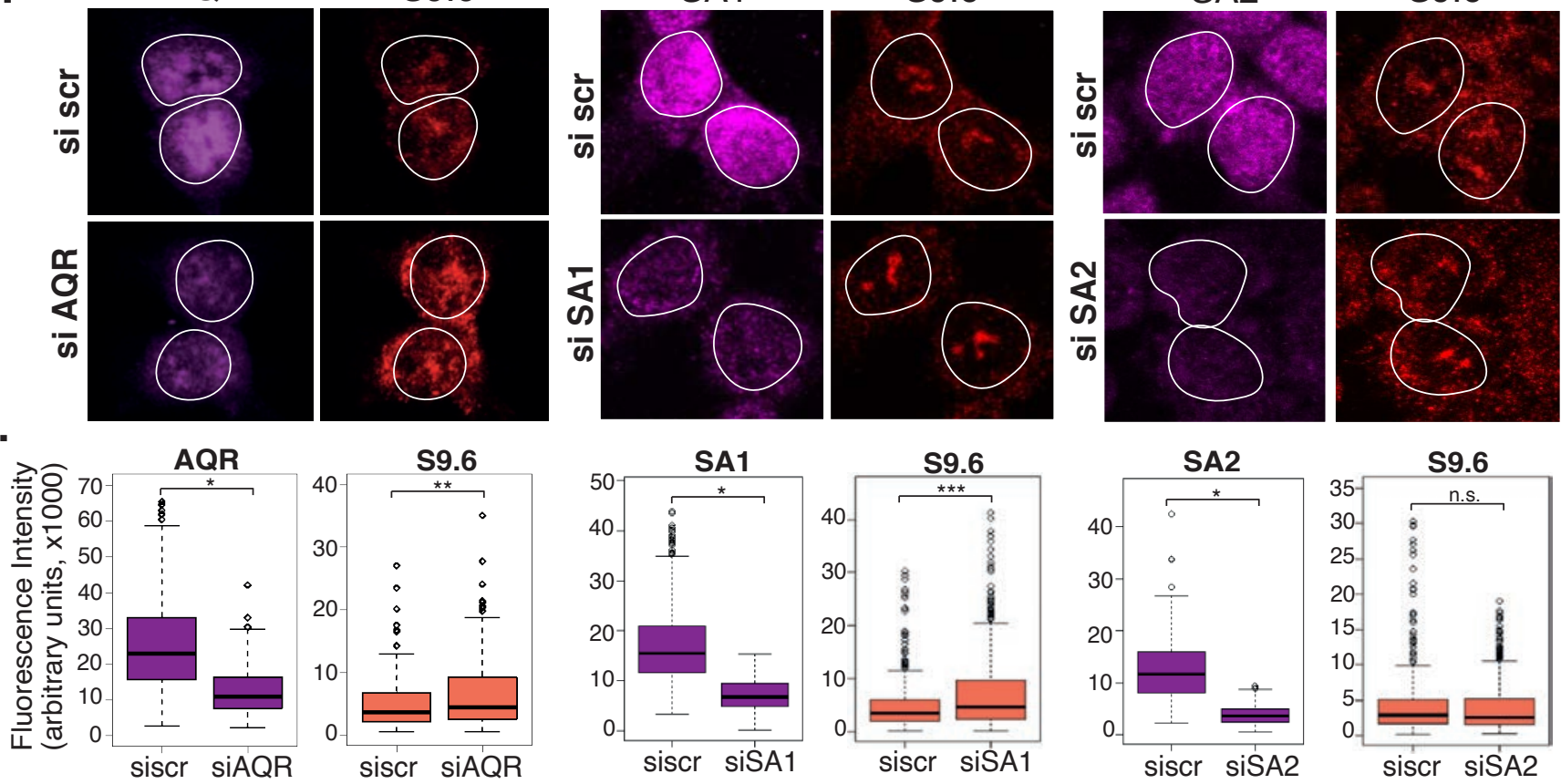

59.6

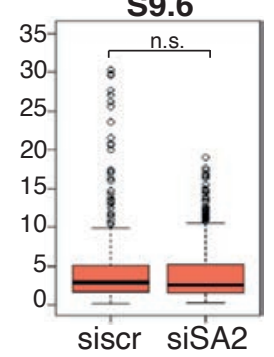


bioRxiv preprint doi: https://doi.org/10.1101/2021.02.20.432055; this version posted February 21, 2021. The copyright holder for this

preprint (which was not certified by peer review) is the author/funder, who has granted bioRxiv a license to display the preprint in

Figure 5.

\section{a.}

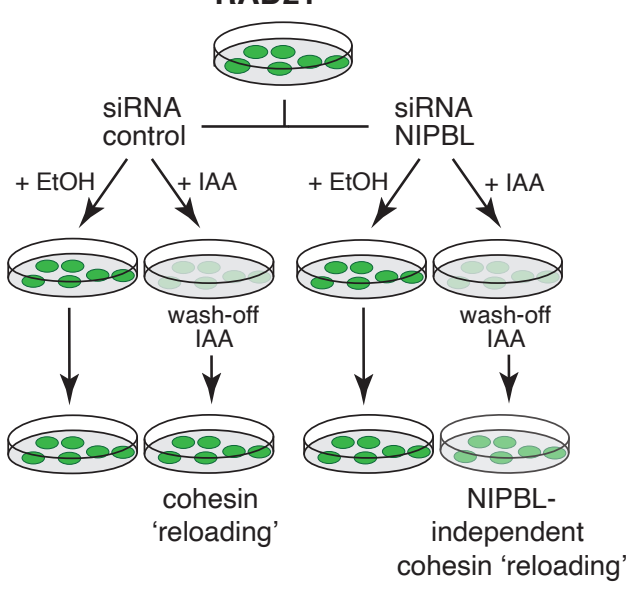

b.

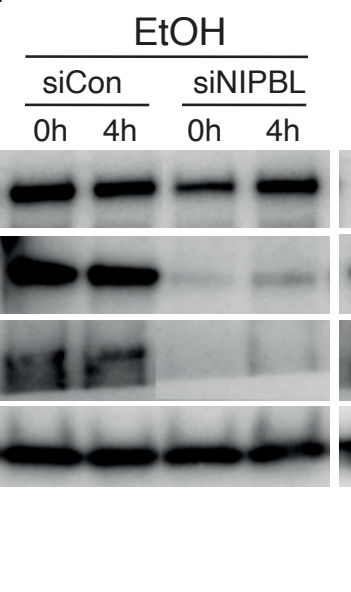

c.

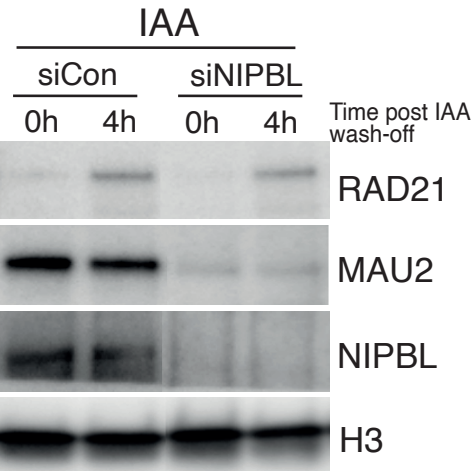
$\square$ siNIPBL Oh

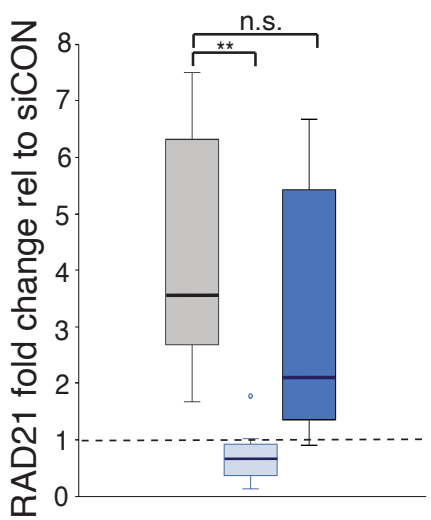

d.

$\mathrm{EtOH}$

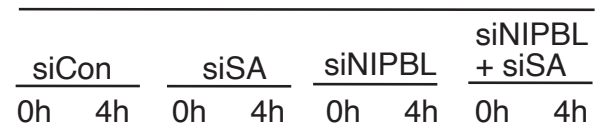

IAA

\begin{tabular}{|c|c|c|c|}
\hline siCon & siSA & siNIPBL & $\begin{array}{l}\text { siNIPBL } \\
+ \text { siSA } \\
\end{array}$ \\
\hline Oh $\quad 4 h$ & Oh $\quad 4 h$ & Oh $\quad 4 h$ & Oh $4 \mathrm{~h}$ \\
\hline
\end{tabular}

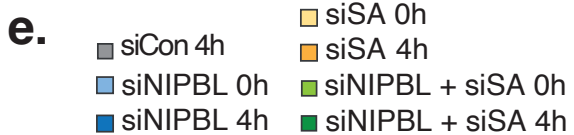

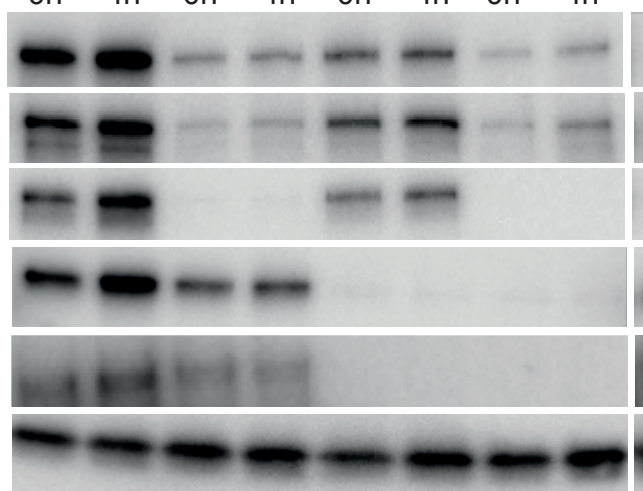

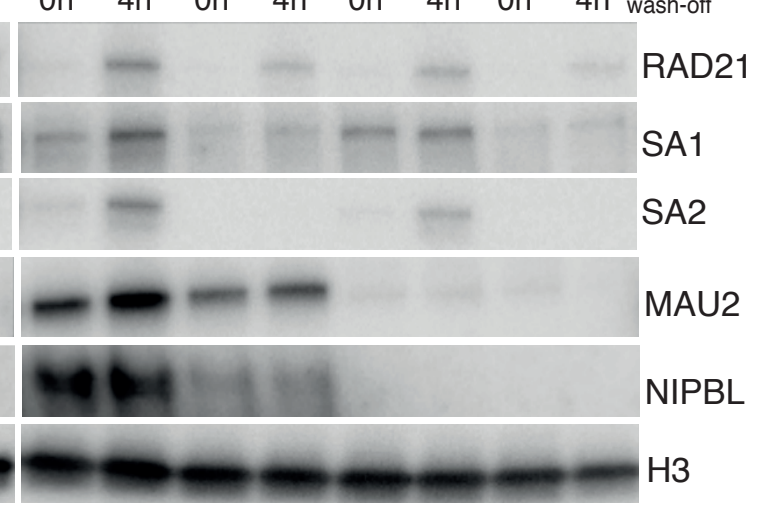
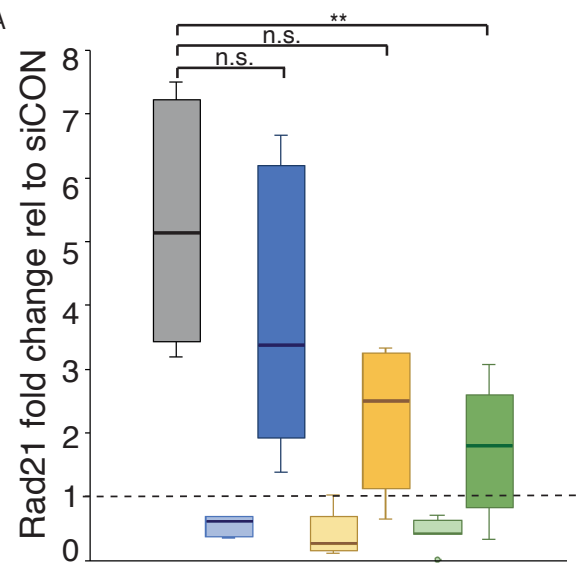

f.

\begin{tabular}{|c|c|c|c|}
\hline \multirow{2}{*}{ siCon } & siNIPBL & siAQR & $\begin{array}{l}\text { siNIPBL } \\
+ \text { siAQR } \\
\end{array}$ \\
\hline & $\mathrm{Oh} \quad 4 \mathrm{~h}$ & Oh $\quad 4 \mathrm{~h}$ & Oh $\quad 4 \mathrm{~h}$ \\
\hline & $-m$ & -1 & $-m$ \\
\hline
\end{tabular}

$\stackrel{\text { siCon }}{\text { on }}$

IAA

\begin{tabular}{|c|c|c|}
\hline siNIPBL & siAQR & $\begin{array}{l}\text { siNIPBL } \\
+ \text { siAQR }\end{array}$ \\
\hline Oh $4 \mathrm{~h}$ & Oh $4 \mathrm{~h}$ & Oh \\
\hline
\end{tabular}

Time post IAA

RAD21

RAD21*

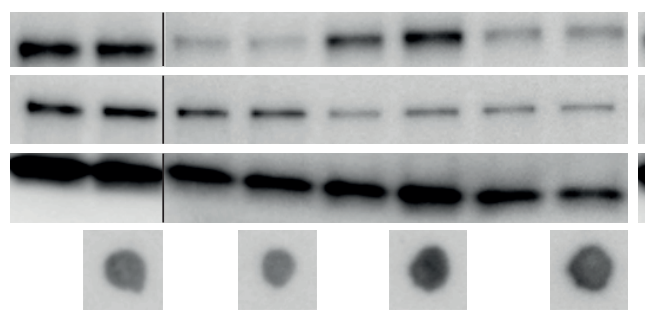

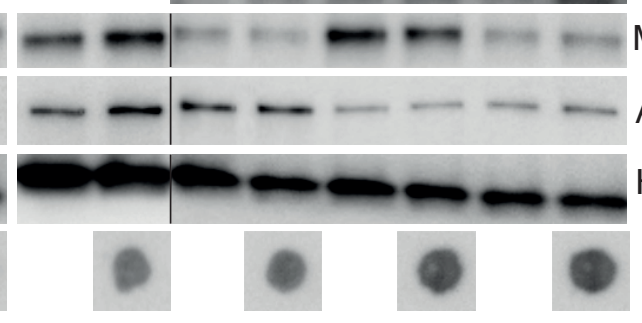

MAU2

AQR

H3

S9.6 g.

sicon $\square$ siAQR

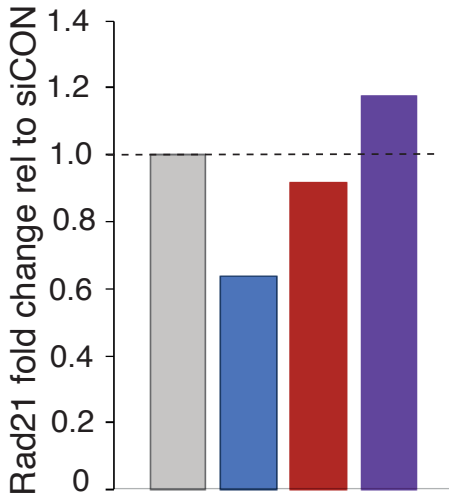

